TeChnical Report CIT-CDS 04-003

Submitted to Automatica: January 26, 2004

\title{
Distributed Receding Horizon Control with Application to Multi-Vehicle Formation Stabilization*
}

\author{
William B. Dunbarł Richard M. Murray \\ Engineering and Applied Science, MS 107-81 \\ California Institute of Technology \\ Pasadena, CA, USA, 91125
}

\begin{abstract}
We consider the control of interacting subsystems whose dynamics and constraints are uncoupled, but whose state vectors are coupled non-separably in a single centralized cost function of a finite horizon optimal control problem. For a given centralized cost structure, we generate distributed optimal control problems for each subsystem and establish that the distributed receding horizon implementation is asymptotically stabilizing. The communication requirements between subsystems with coupling in the cost function are that each subsystem obtain the previous optimal control trajectory of those subsystems at each receding horizon update. The key requirements for stability are that each distributed optimal control not deviate too far from the previous optimal control, and that the receding horizon updates happen sufficiently fast. The theory is applied in simulation for stabilization of a formation of vehicles.
\end{abstract}

Keywords: receding horizon control, model predictive control, distributed control, decentralized control, large scale systems, multi-vehicle formations, coordinated robots.

* Partial support for this work was provided by the DARPA SEC program under grant number F3361598-C-3613 and by AFOSR grant number F49620-01-1-0361.

${ }^{\dagger}$ Corresponding author. Email: dunbar@cds.caltech.edu, Fax: 001-626-796-8914 


\section{Introduction}

We are interested in the control of a set of dynamically decoupled subsystems that are required to perform a cooperative task. An example of such a situation is a group of vehicles cooperatively converging to a desired formation, as explored in Olfati-Saber et al [23], Dunbar and Murray [10], Ren and Beard [25], and Leonard and Fiorelli [17]. One control approach that accommodates a general cooperative objective is receding horizon control. In receding horizon control, or model predictive control, the current control action is determined by solving on-line, at each sampling instant, a finite horizon open-loop optimal control problem. Each optimization yields an open-loop optimal control trajectory and the initial portion of the trajectory is applied to the system until the next sampling instant. A survey of receding horizon control is given by Mayne et al [18]. For the problem of interest here, the cooperation between subsystems can be incorporated in the optimal control problem by including terms in the cost function that depend on their respective state vectors, as is done in [10] and [23]. It is presumed that at least some of the terms that couple states of cooperating subsystems are non-separable, i.e., not additively separable. Otherwise, the subsystems would not be directly cooperating, in the sense that their optimal controls are not directly influenced by the state of the other subsystem. Henceforth, we refer to each subsystem as an agent and any two agents that are cooperating are referred to as neighbors. Thus, neighbors have a non-separable term coupling their states in the single, centralized cost function. Aside from being able to handle the cooperative performance objective, the receding horizon control approach is particularly useful when the individual subsystems are also required to satisfy state and control constraints, as is the case in general for vehicles.

A drawback of the receding horizon control approach to our problem is that currently only a centralized solution and implementation can guarantee asymptotic stability theoretically. However, a distributed solution to the problem is desirable, for autonomy of the individual subsystems and for potential scalability and improved tractability of the approach. In that case, each agent would be assigned its own optimal control problem, implemented in a distributed receding horizon fashion.

Previous work on distributed receding horizon control include Jia and Krogh [14], Motee and Sayyar-Rodsaru [22] and Acar [1]. In all of these papers, the cost is quadratic and separable, while the dynamics are discrete-time, linear, time-invariant and coupled. Further, state and input constraints are not included, aside from a stability constraint in [14] that permits state information exchanged between the agents to be delayed by one update period. In another work, Jia and Krogh [15] solve a min-max problem for each agent, where again coupling comes in the dynamics and the neighboring agent states are treated as bounded disturbances. Stability is obtained by contracting each agents state constraint set at each sample period, until the objective set is reached. As such, stability does not depend on information updates with neighboring agents, although such updates may improve performance. More recently, Keviczky et al [16] have formulated a distributed model predictive scheme where each agent optimizes locally for itself and every neighbor at each update. By this formulation, feasibility becomes difficult to ensure, and no proof of stability is provided. The authors also consider a hierarchical scheme, similar to that in [19], where the scheme 
depends on a particular interconnection graph structure (e.g., no cycles are permitted).

In this paper, we start with an asymptotically stabilizing centralized receding horizon control law, based on the problem formulation and results in the dissertation of Chen [5], which is summarized in [6]. The performance objective is relevant for a multi-vehicle formation stabilization problem. The centralized integrated cost is then decomposed to define distributed integrated costs, and asymptotic stability is proven under stated conditions. Key requirements for stability are that the receding horizon updates happen sufficiently fast, and each distributed optimal control trajectory is required to not deviate too far from the previous optimal trajectory, over the optimized horizon time. We should emphasize that the multi-vehicle formation stabilization problem is simply a venue. In other problems where the centralized integrated cost can be decomposed in the same way, namely such that the summation of the distributed costs recovers the centralized cost, the approach is applicable. With slight modification to the theory in this paper, the dynamics of the individual subsystems need not be linear or homogeneous, i.e., all subsystems could have different, nonlinear dynamics. Such extensions are worked out elsewhere [9].

In our distributed approach, no communication is required between agents while the distributed optimal control problems are being solved. This is an advantage over parallelization methods [3], where every distributed optimization must communicate with neighboring optimizations while iterating. Thus, the approach here would incur less computational and communication delay effects than an approach using receding horizon control with parallelization methods. On the other hand, parallelization can guarantee convergence to the centralized solution, whereas the distributed receding horizon controller here, while stabilizing, will perform differently in general than the centralized receding horizon controller.

The organization of the paper is as follows. Section 2 defines the control objective and cost function used in the optimal control problem. The cost function is relevant for multiple vehicle formation stabilization. We note that the stability results are facilitated by this choice of cost, but the results hold for any cost with a similar decomposable structure. Section 3 defines the optimal control problem and reviews requirements for asymptotic stability of the centralized receding horizon control law. Section 4 details the distributed receding horizon implementation and a proof of asymptotic stability. Simulation results of a multi-vehicle formation are then given in Section 5. Finally, Section 6 discusses conclusions and extensions.

\section{Formation Stabilization Objective}

In this section, we present the system dynamics and constraints and define the control objective. To facilitate the analysis, we consider only linear dynamics here, although the nonlinear case is treated elsewhere [9].

We wish to stabilize a group of agents toward a common objective in a cooperative way using receding horizon control. Each agent is assumed to have dynamics, described by an ordinary differential equation, completely decoupled from all other agents. Specifically, for

$i=1, \ldots, N_{a}$ agents, the state and control of agent $i$ are $z_{i}(t)=\left(q_{i}(t), \dot{q}_{i}(t)\right) \in \mathbb{R}^{2 n}$ and 
$u_{i}(t) \in \mathbb{R}^{n}$, respectively, and the dynamics are given by

$$
\begin{aligned}
& \dot{z}_{i}(t)=A_{i} z_{i}(t)+B_{i} u_{i}(t), \quad t \geq 0, \quad z_{i}(0) \text { given } \\
& \text { where } \quad A_{i}=\left[\begin{array}{cc}
0 & I_{(n)} \\
0 & 0
\end{array}\right], \quad B_{i}=\left[\begin{array}{c}
0 \\
I_{(n)}
\end{array}\right] .
\end{aligned}
$$

The matrix $I_{(n)}$ is the identity matrix of dimension $n$. Each agent $i$ is also subject to the input and state constraints

$$
u_{i}(t) \in \mathcal{U}, \quad z_{i}(t) \in \mathcal{Z}, \quad t \geq 0 .
$$

An admissible control is any piecewise, right-continuous function $u_{i}(\cdot):[0, T] \rightarrow \mathcal{U}$, for any $T \geq 0$, such that given an initial state $z_{i}(0) \in \mathcal{Z}$, the control generates the state trajectory $z_{i}\left(t ; z_{i}(0)\right) \in \mathcal{Z}$ for all $t \in[0, T]$.

The set $\mathcal{Z}^{N}$ is the $N$-times Cartesian product $\mathcal{Z} \times \cdots \times \mathcal{Z}$. Concatenating the states and inputs into vectors as $q=\left(q_{1}, \ldots, q_{N_{a}}\right), \dot{q}=\left(\dot{q}_{1}, \ldots, \dot{q}_{N_{a}}\right), z=\left(z_{1}, \ldots, z_{N_{a}}\right) \in \mathcal{Z}^{N_{a}}$ and $u=\left(u_{1}, \ldots, u_{N_{a}}\right) \in \mathcal{U}^{N_{a}}$, the dynamics are equivalently

$$
\dot{z}(t)=A z(t)+B u(t), \quad t \geq 0, \quad z(0) \text { given, }
$$

where $A=\operatorname{diag}\left(A_{1}, \ldots, A_{N_{a}}\right), B=\operatorname{diag}\left(B_{1}, \ldots, B_{N_{a}}\right)$. Define the invertible map $U: \mathbb{R}^{2 n N_{a}} \rightarrow$ $\mathbb{R}^{2 n N_{a}}$ as

$$
\left[\begin{array}{c}
q \\
\dot{q}
\end{array}\right]=U z .
$$

Note that $U$ is a unitary matrix, so $(U)^{T} U=I$.

Definition 1. The control objective is to cooperatively asymptotically stabilize all agents to $z^{c}=\left(z_{1}^{c}, \ldots, z_{N_{a}}^{c}\right) \in \mathcal{Z}^{N_{a}}$, an equilibrium point of equation (1), with equilibrium control equal to zero.

The cooperation is achieved by the minimization of the cost function defined below. The control objective for each agent $i$ is thus to stabilize to $z_{i}^{c}$ while cooperating with neighboring agents. The position values at $z^{c}$ are denoted $q^{c}=\left(q_{1}^{c}, \ldots, q_{N_{a}}^{c}\right)$, and the equilibrium velocity is clearly zero.

Assumption 1. The following holds:

(i) $\mathcal{U} \subset \mathbb{R}^{n}$ is compact, convex and contains the origin in its interior, and $\mathcal{Z} \subseteq \mathbb{R}^{2 n}$ is convex, connected and contains $z_{i}^{c}$ in its interior, for every $i=1, \ldots, N_{a}$;

(ii) each agent $i$ can measure the full state $z_{i}$, there is no uncertainty, and computational time is negligible compared to the evolution of the closed-loop dynamics.

Remark 1. In the absence of constraints, linear quadratic optimal control could be used to meet the cooperative control objective. Convexity of $\mathcal{U}$ is related to existence of solutions to the optimization problem that will be defined, referring the reader to section 4.3 of [5] and references therein for details. Convexity of both $\mathcal{U}$ and $\mathcal{Z}$ is relevant in guaranteeing that the closed-loop system will have nominal robustness properties [13]. 
The multiple vehicle formation is here defined by a set of relative vectors that connect the desired locations of the vehicles. The desired formation can in turn be viewed as a graph, as in $[11,25]$. For example, consider a desired formation of vehicles in Figure 1, where the position components of $q_{i}$ are denoted $\left(x_{i}, y_{i}\right) \in \mathbb{R}^{2}$. The left figure shows the
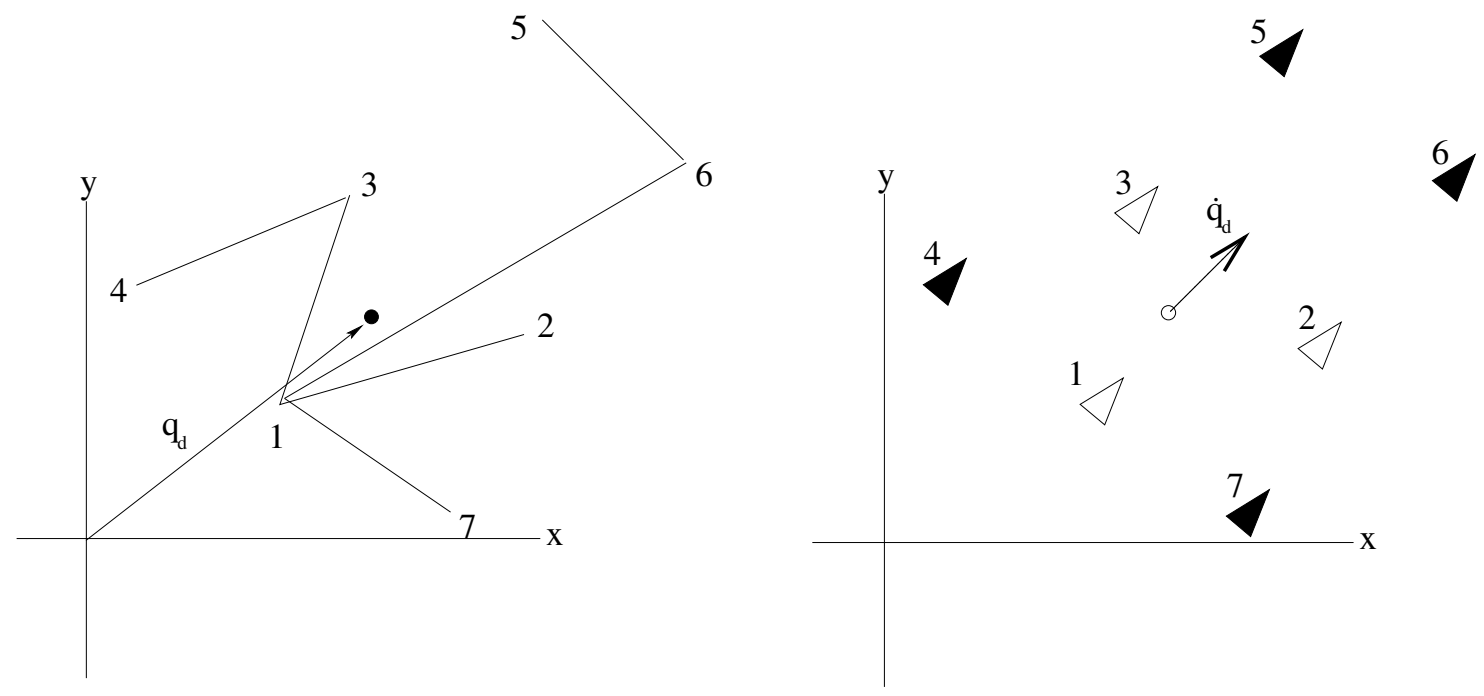

Figure 1: Seven vehicle formation: vector structure on the left, and resulting formation on the right.

vector structure associated with the formation. The numbers correspond to vehicle identity and a line segment between two numbers is a two dimensional relative vector. The dot in the center of the figure is the center of geometry of vehicles 1,2 and 3. Given a desired location for this center of geometry, and the relative vectors between vehicles as shown, this formation designates a globally unique location for each of the vehicles. To generalize, a formation of $N_{a}$ vehicles is uniquely defined given $N_{a}-1$ relative vectors, such that each vehicle is at one end of at least one vector, and 1 vector (denoted $q_{d}$ ) designating a desired center of geometry location for a subset of the vehicles. The vehicles used to relate to $q_{d}$ are called the core vehicles, consistent with the definition in [23]. The figure on the right shows the associated vehicle formation, where the core vehicles are denoted by white triangles, and all other vehicles are denoted by black triangles. The tracking objective is being achieved for some formation path $\mathbb{R} \ni t \mapsto\left(q_{d}(t), \dot{q}_{d}(t)\right) \in \mathbb{R}^{4}$.

We now generalize the description of the formation as a graph. The vector formation graph is defined as $\mathcal{G}=(\mathcal{V}, \mathcal{E})$, where $\mathcal{V}=\left\{1,2, \ldots, N_{a}\right\}$ is the set of vehicles and $\mathcal{E} \subset \mathcal{V} \times \mathcal{V}$ is the set of relative vectors between vehicles, where an edge in the graph is an ordered pair $(i, j) \in \mathcal{E}$ for every relative vector between vehicles $i, j \in \mathcal{V}$. All graphs considered are undirected, so $(i, j) \in \mathcal{E} \Rightarrow(j, i) \in \mathcal{E}$. Two vehicles $i$ and $j$ are called neighbors if $(i, j) \in \mathcal{E}$. The set of neighbors of agent $i$ is denoted $\mathcal{N}_{i} \subset \mathcal{V}$. In addition, although any two core vehicles may not have a relative vector between them in the formation, we consider all core vehicles to be neighbors of one another, as they are all coupled through the tracking 
objective.

Let $\mathcal{E}_{0}$ denote an orientation of the set $\mathcal{E}$, where $\mathcal{E}_{0} \subset \mathcal{E}$ contains one and only one of the two permutations of $(i, j)$, for all $(i, j) \in \mathcal{E}$. Also, without loss of generality, we take the core vehicles to be 1, 2 and 3 , which is the case in Figure 1 . As stated, although $(2,3) \notin \mathcal{E}$, $3 \in \mathcal{N}_{2}$ and $2 \in \mathcal{N}_{3}$ since 2 and 3 are core vehicles and thus coupled through the tracking objective.

Assumption. The undirected vector formation graph $\mathcal{G}$ is connected.

If the formation graph is not connected, there exists a vehicle whose desired location is not uniquely specified by the graph, in addition, the cost function that we define would additively separate into more that one coupled cost function. For a connected graph and resulting cost function defined below, the centralized receding horizon control law involves a single coupled optimal control problem that will be given in the next section. The connectivity assumption clearly holds for the example in Figure 1.

Remark 2. When the minimal number of relative vectors are used to define the formation, $\left|\mathcal{E}_{0}\right|=N_{a}-1$. It may be that more vectors are added to the formation description, provided they are consistent with the existing vectors, as described below. Generally, we shall denote $\left|\mathcal{E}_{0}\right|=M, M \geq N_{a}-1$. In graph theory, assuming the graph is connected implies $M \geq N_{a}-1$ $[4]$.

Let $e_{1}, \ldots, e_{M}$ denote an ordering of the elements of $\mathcal{E}_{0}$. Also, the tail of the edge $e_{i}$, denoted $t\left(e_{i}\right)$, is the first element in the corresponding ordered pair and the head of the vector $h\left(e_{i}\right)$ is the second element. In the case of Figure 1, let

$$
\mathcal{E}_{0}=\left\{e_{1}, e_{2}, e_{3}, e_{4}, e_{5}, e_{6}\right\}=\{(1,2),(1,3),(1,6),(1,7),(3,4),(5,6)\} .
$$

For example, we have $t\left(e_{3}\right)=1$ and $h\left(e_{3}\right)=6$.

Definition 2. The desired relative vector between any two neighbors $i$ and $j$ is denoted $d_{i j} \in \mathbb{R}^{n}$, where it is understood that $q_{i}^{c}+d_{i j}=q_{j}^{c}$. All desired relative vectors are constant vectors, in length and orientation, and satisfy the following consistency conditions:

- For all $(i, j) \in \mathcal{E}, d_{i j}=-d_{j i}$.

- When $M>N_{a}-1$, if $(i, j),(j, l)$ and $(i, l)$ are in $\mathcal{E}$, then $d_{i j}+d_{j l}=d_{i l}$.

Definition 3. Given an admissible oriented formation graph $\mathcal{G}=\left(\mathcal{V}, \mathcal{E}_{0}\right)$ and a formation path, the formation vector $F=\left(f_{1}, \ldots, f_{M+1}\right) \in \mathbb{R}^{n(M+1)}$ has components $f_{l} \in \mathbb{R}^{n}$ defined as

$$
\begin{gathered}
f_{l}=q_{i}-q_{j}+d_{i j}, \quad \text { where } i=t\left(e_{l}\right), j=h\left(e_{l}\right), \forall l=1, \ldots, M, \\
f_{M+1}=q_{\Sigma}-q_{d}, \quad q_{\Sigma}=\frac{1}{3}\left(q_{1}+q_{2}+q_{3}\right) .
\end{gathered}
$$


For a stabilization objective, $\dot{q}_{d}(t)=0, \forall t \in \mathbb{R}$, and to be compatible with the control objective, $q_{d}=\left(q_{1}^{c}+q_{2}^{c}+q_{3}^{c}\right) / 3$. Clearly, the vehicles are in formation when $F \equiv 0$. Write the linear mapping from $q$ to $F$ as:

$$
F=G q+\hat{d}, \quad G^{T}=\left[C_{(n)} V\right] .
$$

The vector $\hat{d}=\left(\ldots, d_{i j}, \ldots,-q_{d}\right)$ has the ordering of the vectors $d_{i j}$ consistent with the definition of $F$. The matrix $V^{T}=\left[\begin{array}{lll}V_{1} & \ldots & V_{N_{a}}\end{array}\right] \in \mathbb{R}^{n \times n N_{a}}$ has elements $V_{i} \in \mathbb{R}^{n \times n}$ defined as

$$
V_{i}=\left\{\begin{array}{ll}
\frac{1}{3} I_{(n)}, & \text { if } \mathrm{i}=1,2,3 \\
0, & \text { otherwise, }
\end{array} .\right.
$$

The matrix $C_{(n)} \in \mathbb{R}^{n\left(N_{a} \times M\right)}$ is related to the incidence matrix $C \in \mathbb{R}^{N_{a} \times M}$, where the elements of $C=\left[c_{i j}\right]$ are defined in terms of the elements of the oriented edge set $\mathcal{E}_{0}$ as

$$
c_{i j}=\left\{\begin{array}{cl}
+1, & \text { vertex } i=t\left(e_{j}\right) \\
-1, & \text { vertex } i=h\left(e_{j}\right) . \\
0, & \text { otherwise }
\end{array}\right.
$$

The matrix $C_{(n)}$ is defined by replacing each element of $C$ with that element multiplied by $I_{(n)}$. The incidence matrix for the example in Figure 1 is

$$
C=\left[\begin{array}{rrrrrr}
1 & 1 & 1 & 1 & 0 & 0 \\
-1 & 0 & 0 & 0 & 0 & 0 \\
0 & -1 & 0 & 0 & 1 & 0 \\
0 & 0 & 0 & 0 & -1 & 0 \\
0 & 0 & 0 & 0 & 0 & 1 \\
0 & 0 & -1 & 0 & 0 & -1 \\
0 & 0 & 0 & -1 & 0 & 0
\end{array}\right]
$$

In defining the cost function for the optimal control problem, the following proposition is useful.

Proposition 1. The matrix $G$ in equation (2) has full column rank, equal to $\operatorname{dim}(q)=n N_{a}$.

Proof. Since the vector formation graph is connected, the incidence matrix $C$ has rank $\left(N_{a}-1\right)$ [4]. Scaling the entries of $C$ with the identity matrix $I_{(n)}$ implies the rank of $C_{(n)}$ is equal to $n\left(N_{a}-1\right)$. The matrix $V$ in equation (2) has full column rank equal to $n$, and each column vector is linearly independent from the column vectors in $C_{(n)}$. Thus, $G$ has rank $n N_{a}$, which is the column dimension of the matrix.

Remark 3. For the rest of the paper, we shall use the norm $\|z\|$ to denote the Euclidean norm of any vector $z \in \mathbb{R}^{m}$. In cases where $z$ is a curve, we abuse the notation $\|z\|$ to mean $\|z(t)\|$ at some instant of time $t$. 
From the definition of the formation vector, we know that $G q^{c}=-\hat{d}$ and so

$$
\|F\|^{2}=\left(G\left(q-q^{c}\right)\right)^{T} G\left(q-q^{c}\right)=\left\|q-q^{c}\right\|_{G^{T} G}^{2} .
$$

In the following we penalize $\|F\|^{2}$ and $\|\dot{q}\|^{2}$ in the centralized cost function. To define the objective in terms of the state $z$, we have that

$$
\left[\begin{array}{c}
G\left(q-q^{c}\right) \\
\dot{q}
\end{array}\right]=\widehat{G} U\left(z-z^{c}\right), \quad \text { where } \quad \widehat{G}=\left[\begin{array}{cc}
G & 0 \\
0 & I_{\left(n N_{a}\right)}
\end{array}\right]
$$

As a result, we have

$$
\|F\|^{2}+\|\dot{q}\|^{2}=\left\|z-z^{c}\right\|_{\widehat{G}^{T} \widehat{G}}^{2} .
$$

In the next section, the optimal control problem associated with the multiple vehicle formation stabilization objective is defined for receding horizon control.

\section{Receding Horizon Control}

In this section, we give the receding horizon control law that achieves the cooperative control objective, implemented in a centralized fashion. The centralized integrated cost function of interest is

$$
L(z, u)=\sum_{(i, j) \in \mathcal{E}_{0}} \omega\left\|q_{i}-q_{j}+d_{i j}\right\|^{2}+\omega\left\|q_{\Sigma}-q_{d}\right\|^{2}+\nu\|\dot{q}\|^{2}+\mu\|u\|^{2}
$$

with positive weighting constants $\omega, \nu$ and $\mu$. We refer to the term $\omega\left\|q_{\Sigma}-q_{d}\right\|^{2}$ as the tracking cost, although we are concerned with stabilization.

Remark 4. For collision avoidance, an appropriate cost function between any two agents is defined in [23]. Alternatively, to guarantee avoidance, collision avoidance can be cast as a constraint, as in [26]. We do not incorporate any type of collision avoidance in this paper, although coupling constraints between neighboring agents will be discussed in the conclusions.

From the previous section, we also have that

$$
L(z, u)=\left\|z-z^{c}\right\|_{Q}^{2}+\mu\|u\|^{2}, \quad Q=\left[\begin{array}{cc}
\omega G^{T} G & 0 \\
0 & \nu I_{\left(n N_{a}\right)}
\end{array}\right] .
$$

From Proposition 1, $Q$ is positive definite and clearly symmetric. At any time $t$, given $z(t)$ and fixed horizon time $T$, the centralized open-loop optimal control problem is

Problem 1. Find

$$
J^{*}(z(t), T)=\min _{u(\cdot)} J(z(t), u(\cdot), T)
$$


with

$$
J(z(t), u(\cdot), T)=\int_{t}^{t+T}\left\|z(\tau ; z(t))-z^{c}\right\|_{Q}^{2}+\mu\|u(\tau)\|^{2} \mathrm{~d} \tau+\left\|z(t+T ; z(t))-z^{c}\right\|_{P}^{2},
$$

subject to

$$
\left.\begin{array}{l}
\dot{z}(s)=A z(s)+B u(s) \\
u(s) \in \mathcal{U}^{N_{a}} \\
z(s ; z(t)) \in \mathcal{Z}^{N_{a}} \\
z(t+T ; z(t)) \in \Omega(\alpha),
\end{array}\right\} s \in[t, t+T]
$$

where $P=P^{T}>0$ and

$$
\Omega(\alpha):=\left\{z \in \mathbb{R}^{2 n N_{a}}:\left\|z-z^{c}\right\|_{P}^{2} \leq \alpha, \alpha \geq 0\right\} .
$$

The equation (4) is called the terminal constraint, as it is a constraint enforced only at the terminal or end time. Let the first optimal control problem be initialized at some time $t_{0} \in \mathbb{R}$ and let $\delta$ denote the receding horizon update period. The closed-loop system, for which stability is to be guaranteed, is

$$
\dot{z}(\tau)=A z(\tau)+B u_{\text {cent }}^{*}(\tau), \quad \tau \geq t_{0},
$$

where the centralized receding horizon control law is

$$
u_{\text {cent }}^{*}(\tau)=u_{\text {cent }}^{*}(\tau ; z(t)), \tau \in[t, t+\delta], 0<\delta \leq T,
$$

and $u_{\text {cent }}^{*}(s ; z(t)), s \in[t, t+T]$, is the optimal open-loop solution (assumed to exist) to Problem 1 with initial state $z(t)$. The receding horizon control law is defined for all $t \geq t_{0}$ by applying the open-loop optimal solution until each new initial state update $z(t) \leftarrow z(t+\delta)$ is available. This is what we mean when we say a controller is implemented in a "receding horizon fashion", since the optimization horizon is always $T$ seconds ahead of each new update time. The reason to use a sampling period shorter than the open-loop horizon time is that, practically, there is uncertainty and applying only a fraction of the open-loop control before re-sampling and recomputing mitigates the effects of uncertainty. The notation above shows the implicit dependence of the optimal open-loop control $u_{\text {cent }}^{*}(\cdot)$ on the initial state $z(t)$ through the optimal control problem. The optimal open-loop state trajectory is denoted $z_{\text {cent }}^{*}(\tau ; z(t))$. Since Problem 1 is time-invariant, we can set $t=0$ and solve the optimal control problem at each initial state update over the time interval $[0, T]$.

Proof of asymptotic stability of the closed-loop dynamics under the receding horizon control implementation can be established by taking the optimal cost function $J^{*}(\cdot)$ as a Lyapunov function.

Definition 4. A feasible control is any admissible control such that all state constraints in Problem 1 are satisfied and the optimal cost function is bounded. Let $Z$ denote the set of states for which there exists a feasible control. 
Assumption 2. The following conditions are satisfied:

(i) the largest constant $\alpha>0$ in the terminal constraint (4) is chosen such that $\Omega(\alpha) \subseteq$ $\mathcal{Z}^{N_{a}}$ and the linear state feedback $u=K\left(z-z^{c}\right)$ and the positive-definite, symmetric terminal cost $P$ satisfy

$$
\left.\begin{array}{l}
(A+B K)^{T} P+P(A+B K)=-\left(Q+\mu K^{T} K\right) \\
K\left(z-z^{c}\right) \in \mathcal{U}^{N_{a}}, \quad \forall z \in \Omega(\alpha)
\end{array}\right\}
$$

(ii) the optimal solution to Problem 1 exists and is numerically obtainable for all $z \in Z$.

Theorem 1. [6, Theorem 1] Under Assumptions 1 and 2, for any $\delta \in(0, T], z^{c}$ is an asymptotically stable equilibrium point of the closed-loop system (5) with region of attraction $Z$.

The stability result in [6] only requires that Problem 1 be feasible at initialization, rather than requiring the optimal solution at each update. Also, $\delta$ is required to be sufficiently small since the authors consider quantization errors in the numerical implementation of the receding horizon control law.

So far, we have detailed the conditions required for asymptotic stability of the centralized receding horizon control law. In the next section, $N_{a}$ optimal control problems are defined for a distributed receding horizon implementation. It will be proven that, for sufficiently fast updates (small $\delta$ ), the distributed receding horizon control laws are asymptotically stabilizing.

\section{Distributed Receding Horizon Control}

In this section, a distributed receding horizon control law is defined. We first introduce some useful notation and define $N_{a}$ separate optimal control problems, that are solved and implemented in a distributed receding horizon fashion. Next, we analyze the stability of the closed-loop system. Finally, we comment on alternative formulations.

\subsection{Distributed Optimal Control Problems}

In the centralized integrated cost, the non-separable terms $\left\|q_{i}-q_{j}+d_{i j}\right\|^{2}$, for all $(i, j) \in \mathcal{E}_{0}$, as well as the tracking term $\left\|q_{\Sigma}-q_{d}\right\|^{2}$, couple the states of neighboring agents. Recall that the set of neighbors of each agent $i$ is denoted $\mathcal{N}_{i}$. In some cases, it will be easier to denote the set of neighbors $\mathcal{N}_{i}$ as $-i$ and both shall be used interchangeably.

Let $z_{-i}=\left(z_{j_{1}}, \ldots, z_{j_{\left|\mathcal{N}_{i}\right|}}\right)$ denote the vector of states of the neighbors of $i$, i.e., $j_{k} \in \mathcal{N}_{i}, k=$ $1, \ldots,\left|\mathcal{N}_{i}\right|$, where the ordering of the states is arbitrary but fixed. Also, let $q_{-i}=\left(q_{j_{1}}, \ldots, q_{j_{\left|\mathcal{N}_{i}\right|} \mid}\right)$ and $u_{-i}=\left(u_{j_{1}}, \ldots, u_{j_{\left|\mathcal{N}_{i}\right|}}\right)$, where the ordering is consistent with $z_{-i}$. 
Definition 5. The distributed integrated cost in the optimal control problem for any agent $i=1, \ldots, N_{a}$ is defined as

$$
\begin{gathered}
L_{i}\left(z_{i}, z_{-i}, u_{i}\right)=L_{i}^{z}\left(z_{i}, z_{-i}\right)+\gamma \mu\left\|u_{i}\right\|^{2}, \\
\text { where } L_{i}^{z}\left(z_{i}, z_{-i}\right)=\gamma\left[\sum_{j \in \mathcal{N}_{i}}\left\{\frac{\omega}{2}\left\|q_{i}-q_{j}+d_{i j}\right\|^{2}\right\}+\nu\left\|\dot{q}_{i}\right\|^{2}+L_{d}(i)\right], \\
\gamma>1, \quad L^{d}(i)= \begin{cases}\frac{\omega}{3}\left\|q_{\Sigma}-q_{d}\right\|^{2}, & i=1,2,3 \\
0, & \text { otherwise. }\end{cases}
\end{gathered}
$$

Thus, $\sum_{i=1}^{N_{a}} L_{i}\left(z_{i}, z_{-i}, u_{i}\right)=\gamma L(z, u)=\gamma\left[\left\|z-z^{c}\right\|_{Q}^{2}+\mu\|u\|^{2}\right]$.

From the definition, the distributed integrated cost for any agent $i$ includes: one-half of each relative vector penalty coupling $i$ with each neighbor $j \in \mathcal{N}_{i}$, the velocity and control penalties for $i$, and, if $i$ is one of the three core vehicles (1, 2 or 3), one-third of the tracking penalty. In addition, all terms are multiplied by a common factor $\gamma$, a constant greater than one. In the proof of stability, the key structure is that the sum of the distributed integrated costs equals the centralized cost multiplied by $\gamma$. For any problem where the centralized cost can be decomposed in the same way, and the other stated assumptions hold, the stability results that follow are applicable.

Remark 5. The stability results that follow do not depend on equal weighting of terms between neighboring agents. What is required is that the distributed integrated costs sum up to be the centralized cost, multiplied by a factor $(\gamma)$ greater than one. The weighting will of course affect the performance of the closed-loop system, so making the weights lop-sided would result in one agent reacting more to the term than the corresponding neighbor. Note that in the limit that one agent takes the entire term, while the other ignores the term, we have a leader-follower effect.

At each update of the distributed receding horizon control laws, every agent

- senses its own current state and senses or receives the current state of its neighbors, and

- computes the optimal control trajectory, comparing it to an assumed control trajectory and based on some assumed control trajectories for its neighbors.

Prior to the next receding horizon update, every agent

- implements the current optimal control trajectory,

- computes the next assumed control trajectory, to be used at the next update,

- transmits the assumed trajectory to all of its neighbors and receives the assumed control trajectories from each neighbor. 
Implicit in the procedure above is that the assumed control for each agent $i$ is consistent in every optimization problem that it occurs, i.e., in the optimal control problem for agent $i$ and for each neighbor $j \in \mathcal{N}_{i}$. Before defining the computation for the optimal and assumed control trajectories, we introduce some notation.

Definition 6. Consider a common interval of time $[t, t+T]$ in the optimal control problem for every agent $i=1, \ldots, N_{a}$. Associated with the initial state $z_{i}(t)$, we denote:

applied control $u_{i}\left(\cdot ; z_{i}(t)\right)$ the control being optimized in the problem and applied to the system over the subinterval $[t, t+\delta]$;

assumed control $\hat{u}_{i}\left(\cdot ; z_{i}(t)\right)$ the control to which the optimized control is compared and which all neighbors assume $i$ is employing over the interval.

The state trajectories corresponding to the applied and assumed controls are denoted $z_{i}\left(\cdot ; z_{i}(t)\right)$ and $\hat{z}_{i}\left(\cdot ; z_{i}(t)\right)$, respectively. For each agent $i$, given the current state $z_{j}(t)$ and assumed control $\hat{u}_{j}\left(s ; z_{j}(t)\right), s \in[t, t+T]$, of every neighbor $j \in \mathcal{N}_{i}$, the assumed state trajectory $\hat{z}_{j}\left(s ; z_{j}(t)\right), s \in[t, t+T]$, is computed using the dynamic model for that agent. An important point is that the initial condition of every assumed state trajectory is equal to the actual state value of the corresponding agent at that time, that is

$$
\hat{z}_{i}\left(t ; z_{i}(t)\right)=z_{i}(t)
$$

for every $i=1, \ldots, N_{a}$. To be consistent with the notation $z_{-i}$, let $\hat{z}_{-i}\left(\cdot ; z_{-i}(t)\right)$ and $\hat{u}_{-i}\left(\cdot ; z_{-i}(t)\right)$ be the vector of assumed neighbor states and controls, respectively, of agent $i$. With consistent initial conditions then we also have that $\hat{z}_{-i}\left(t ; z_{-i}(t)\right)=z_{-i}(t)$.

The distributed optimal control problems are now defined. Denote the receding horizon update times as $t_{k}=t_{0}+\delta k$, where $k \in \mathbb{N}=\{0,1,2, \ldots\}$. Common to each problem, we are given the constant $\gamma \in(1, \infty)$ from Definition 5 , update period $\delta \in(0, T)$ and fixed horizon time $T$. Conditions will be placed on the update period $\delta$ in the next section to guarantee stability of the closed-loop system. The collection of distributed open-loop optimal control problems is

Problem 2. For every agent $i=1, \ldots, N_{a}$ and at any update time $t_{k}$, given $z_{i}\left(t_{k}\right), \hat{z}_{-i}\left(t_{k} ; z_{-i}\left(t_{k}\right)\right)=$ $z_{-i}\left(t_{k}\right)$, and $\hat{u}_{i}\left(s ; z_{i}\left(t_{k}\right)\right)$ and $\hat{u}_{-i}\left(s ; z_{-i}\left(t_{k}\right)\right)$ for all $s \in\left[t_{k}, t_{k}+T\right]$, find

$$
J_{i}^{*}\left(z_{i}\left(t_{k}\right), z_{-i}\left(t_{k}\right), T\right)=\min _{u_{i}(\cdot)} J_{i}\left(z_{i}\left(t_{k}\right), z_{-i}\left(t_{k}\right), u_{i}\left(\cdot ; z_{i}\left(t_{k}\right)\right), T\right),
$$

where $J_{i}\left(z_{i}\left(t_{k}\right), z_{-i}\left(t_{k}\right), u_{i}\left(\cdot ; z_{i}\left(t_{k}\right)\right), T\right)$ is equal to

$$
\int_{t_{k}}^{t_{k}+T} L_{i}\left(z_{i}\left(\tau ; z_{i}\left(t_{k}\right)\right), \hat{z}_{-i}\left(\tau ; z_{-i}\left(t_{k}\right)\right), u_{i}\left(\tau ; z_{i}\left(t_{k}\right)\right)\right) \mathrm{d} \tau+\gamma\left\|z_{i}\left(t_{k}+T ; z_{i}\left(t_{k}\right)\right)-z_{i}^{c}\right\|_{P_{i}}^{2}
$$


subject to

$$
\left.\begin{array}{l}
\dot{z}_{i}\left(s ; z_{i}\left(t_{k}\right)\right)=A_{i} z_{i}\left(s ; z_{i}\left(t_{k}\right)\right)+B_{i} u_{i}\left(s ; z_{i}\left(t_{k}\right)\right) \\
\dot{\hat{z}}_{j}\left(s ; z_{j}\left(t_{k}\right)\right)=A_{j} \hat{z}_{j}\left(s ; z_{j}\left(t_{k}\right)\right)+B_{j} \hat{u}_{j}\left(s ; z_{j}\left(t_{k}\right)\right), \forall j \in \mathcal{N}_{i} \\
u_{i}\left(s ; z_{i}\left(t_{k}\right)\right) \in \mathcal{U} \\
z_{i}\left(s ; z_{i}(t)\right) \in \mathcal{Z} \\
\left\|u_{i}\left(s ; z_{i}\left(t_{k}\right)\right)-\hat{u}_{i}\left(s ; z_{i}\left(t_{k}\right)\right)\right\| \leq \delta^{2} \kappa \\
z_{i}\left(t_{k}+T ; z_{i}\left(t_{k}\right)\right) \in \Omega_{i}\left(\varepsilon_{i}\right),
\end{array}\right\} \quad s \in\left[t_{k}, t_{k}+T\right]
$$

given positive constant $\kappa \in(0, \infty), P_{i}=P_{i}^{T}>0$ and where

$$
\Omega_{i}\left(\varepsilon_{i}\right):=\left\{z \in \mathbb{R}^{2 n}:\left\|z-z_{i}^{c}\right\|_{P_{i}}^{2} \leq \varepsilon_{i}, \varepsilon_{i} \geq 0\right\}
$$

As part of the optimal control problem, the applied control for $i$ is constrained to be at most a distance of $\delta^{2} \kappa$ from the assumed control. We refer to this constraint as the control comparison constraint. Naturally, the constraint is a means of enforcing a degree of consistency between what an agent is actually doing and what neighbors believe that agent is doing. The assumed control for each agent, as well as each terminal cost weighting $P_{i}$, will be defined below.

Remark 6. Instead of communicating assumed controls, neighboring agents could compute and transmit the corresponding assumed state, since that is what each distributed optimal control problem depends upon. That would remove the need for the differential equation of each neighbor, simplifying each local optimization problem. We shall discuss this further in Section 4.3.

The optimal solution to each distributed optimal control problem, assumed to exist, is denoted

$$
u_{d i}^{*}\left(\tau ; z_{i}\left(t_{k}\right)\right), \tau \in\left[t_{k}, t_{k}+T\right] .
$$

The closed-loop system, for which stability is to be guaranteed, is

$$
\dot{z}(\tau)=A z(\tau)+B u_{\text {dist }}^{*}(\tau), \quad \tau \geq t_{0}
$$

where the distributed receding horizon control law is

$$
u_{\text {dist }}^{*}\left(\tau ; z\left(t_{k}\right)\right)=\left(u_{d 1}^{*}\left(\tau ; z_{1}\left(t_{k}\right)\right), \ldots, u_{d N_{a}}^{*}\left(\tau ; z_{N_{a}}\left(t_{k}\right)\right)\right),
$$

for $\tau \in\left[t_{k}, t_{k}+\delta\right], 0<\delta<T$ and $k \in \mathbb{N}$. As before, the receding horizon control law is updated when each new initial state update $z\left(t_{k}\right) \leftarrow z\left(t_{k+1}\right)$ is available. The optimal state for agent $i$ is denoted $z_{d i}^{*}\left(\tau ; z_{i}\left(t_{k}\right)\right)$, for all $\tau \in\left[t_{k}, t_{k}+T\right]$. The concatenated vector of the distributed optimal states is denoted

$$
z_{\text {dist }}^{*}\left(\tau ; z\left(t_{k}\right)\right)=\left(z_{d 1}^{*}\left(\tau ; z_{1}\left(t_{k}\right)\right), \ldots, z_{d N_{a}}^{*}\left(\tau ; z_{N_{a}}\left(t_{k}\right)\right)\right)
$$

for all $\tau \in\left[t_{k}, t_{k}+T\right]$. Although we denote the optimal control for agent $i$ as $u_{d i}^{*}\left(\tau ; z_{i}\left(t_{k}\right)\right)$, it is understood that this control is implicitly dependent on the initial state $z_{i}\left(t_{k}\right)$ and the initial states of the neighbors $z_{-i}\left(t_{k}\right)$. 
Assumption 3. The following conditions are satisfied, for every $i=1, \ldots, N_{a}$ :

(i) the positive constants $\varepsilon_{i}>0$ are chosen such that $\Omega_{i}\left(\varepsilon_{i}\right) \subseteq \mathcal{Z}$ and such that an asymptotically stabilizing feedback $u_{i}=K_{i}\left(z_{i}-z_{i}^{c}\right)$ and positive-definite, symmetric matrix $P_{i}$ satisfy

$$
\left.\begin{array}{l}
\left(A_{i}+B_{i} K_{i}\right)^{T} P_{i}+P_{i}\left(A_{i}+B_{i} K_{i}\right)=-\left(Q_{i}+\mu K_{i}^{T} K_{i}\right) \\
K_{i}\left(z_{i}-z_{i}^{c}\right) \in \mathcal{U}, \quad \forall z_{i} \in \Omega_{i}\left(\varepsilon_{i}\right) .
\end{array}\right\}
$$

Moreover, $Q_{i}$ is chosen such that $\widehat{Q}=\operatorname{diag}\left(Q_{1}, \ldots, Q_{N_{a}}\right)$ satisfies $\widehat{Q} \geq Q$, where $Q$ is defined in equation (3);

(ii) at any receding horizon update time, the collection of open-loop optimal control problems in Problem 2 are solved globally synchronously;

(iii) communication of control trajectories between neighboring agents is lossless.

Remark 7. The receding horizon control law is employed for all time after the initialization and the decoupled linear feedbacks $K_{i}$ need not be employed, even after agent $i$ enters $\Omega_{i}\left(\varepsilon_{i}\right)$. The value for $\varepsilon_{i}$ simply determines the size of the set over which the conditions in Assumption 3 are satisfied. It may be desired that an agent switch from the receding horizon controller to the decoupled linear feedbacks once inside the terminal constraint set, known as dual-mode receding horizon control in the literature [20]. Generally, $\varepsilon_{i}$ could be chosen to satisfy the conditions in Assumption 3 and the additional condition

$$
\Omega_{i}\left(\varepsilon_{i}\right) \bigcap \Omega_{j}\left(\varepsilon_{j}\right)=\emptyset, \quad \text { for all } i, j=1, \ldots, N_{a}, i \neq j,
$$

so that once inside $\Omega_{i}\left(\varepsilon_{i}\right)$, any agent $i$ is closer to its objective state than any other agents objective state. In that case, the agents employ the decoupled feedbacks only if they are close enough to their objective state and far enough from any other agents objective state.

Remark 8. Due to condition (ii) above, the distributed receding horizon control laws are not technically decentralized, since a globally synchronous implementation requires centralized clock keeping [3]. However, a locally synchronous, and consequently decentralized, version is also currently being constructed [9].

Although not employed, the decoupled linear feedbacks $K_{i}$ can asymptotically stabilize each agent to its objective state once the agent enters the decoupled terminal constraint set (7). One choice for $Q_{i}$ that would satisfy $\widehat{Q} \geq Q$ is $Q_{i}=\lambda_{\max }(Q) I_{(2 n)}$, where $\lambda_{\max }(Q)$ is the maximum eigenvalue of the symmetric matrix $Q$. Define

$$
\widehat{K}=\operatorname{diag}\left(K_{1}, \ldots, K_{N_{a}}\right), \quad \widehat{P}=\operatorname{diag}\left(P_{1}, \ldots, P_{N_{a}}\right) .
$$

As a consequence of assumption (i) above, $\widehat{P}$ is positive-definite and symmetric, and satisfies

$$
(A+B \widehat{K})^{T} \widehat{P}+\widehat{P}(A+B \widehat{K})=-\left(\widehat{Q}+\mu \widehat{K}^{T} \widehat{K}\right) \leq-\left(Q+\mu \widehat{K}^{T} \widehat{K}\right) .
$$

We now define the initialization procedure for the distributed receding horizon control law, and the assumed control for each agent at each update time. 
Definition 7. (Initialization) Denote time $t_{-1}=t_{0}-\delta$. Solve Problem 2 with initial state $z\left(t_{-1}\right)$, setting $\hat{u}_{i}\left(\tau ; z_{i}\left(t_{-1}\right)\right)=0$ for all $\tau \in\left[t_{-1}, t_{-1}+T\right]$ and every $i=1, \ldots, N_{a}$, and also setting $\kappa=+\infty$. The optimal trajectories are denoted $u_{d i}^{*}\left(\tau ; z_{i}\left(t_{-1}\right)\right)$ and $z_{d i}^{*}\left(\tau ; z_{i}\left(t_{-1}\right)\right)$, for every $i=1, \ldots, N_{a}$. The optimal control $u_{\text {dist }}^{*}\left(\tau ; z\left(t_{-1}\right)\right)$ is applied for $\tau \in\left[t_{-1}, t_{0}\right]$.

At initialization, the control comparison constraint is effectively removed by setting $\kappa$ to a large number. The assumed controls at initialization will have an impact on closedloop performance. If instead the centralized problem were solved at time $t_{-1}$, and the solution disseminated to the agents, the closed-loop performance may be closer to that of the centralized implementation. In the simulation results, we note that the performance is already close to that of the centralized implementation using the initialization procedure as defined above.

Definition 8. (Assumed Control) For each agent $i=1, \ldots, N_{a}$ and for any $k \in \mathbb{N}$, the assumed control $\hat{u}_{i}\left(\cdot ; z_{i}\left(t_{k}\right)\right):\left[t_{k}, t_{k}+T\right] \rightarrow \mathcal{U}$ is defined as follows:

if $z\left(t_{k}\right)=z^{c}$, then $\hat{u}_{i}\left(\tau ; z_{i}\left(t_{k}\right)\right)=0, \quad \tau \in\left[t_{k}, t_{k}+T\right]$,

otherwise $\hat{u}_{i}\left(\tau ; z_{i}\left(t_{k}\right)\right)=\left\{\begin{array}{ll}u_{d i}^{*}\left(\tau ; z_{i}\left(t_{k-1}\right)\right), & \tau \in\left[t_{k}, t_{k-1}+T\right] \\ K_{i}\left(z_{i}^{k}\left(\tau ; z_{d i}^{*}\left(t_{k-1}+T ; z_{i}\left(t_{k-1}\right)\right)\right)-z_{i}^{c}\right), & \tau \in\left[t_{k-1}+T, t_{k}+T\right]\end{array}\right.$,

where $z_{i}^{k}\left(s ; z_{i}^{k}(0)\right)$ is the closed-loop solution to

$$
\dot{z}_{i}^{k}(s)=\left(A_{i}+B_{i} K_{i}\right)\left(z_{i}^{k}(s)-z_{i}^{c}\right), \quad s \geq 0, \quad \text { given } z_{i}^{k}(0) .
$$

The assumed control for agent $i$ at initial time $t_{k}$ is generated and transmitted to each neighbor $j \in \mathcal{N}_{i}$ in the time window $\left[t_{k-1}, t_{k}\right]$.

To state Definition 8 in words, in Problem 2 every agent is assuming all neighbors will continue along their previous optimal path, finishing with the decoupled linear control laws defined in Assumption 3, unless the control objective is met at any update time after initialization. In the latter case, neighbors are assumed to do nothing, i.e., apply zero control. Notice that the communication of control trajectories between neighboring agents is not required to happen instantaneously, but over each receding horizon update time interval.

Remark 9. The test of whether $z\left(t_{k}\right)=z^{c}$ in generating the assumed control is a centralized test. The reason for the test is its use in the proof of Proposition 2 in the next section. We note that the asymptotic stability result in the next section guarantees that only in the limit as $t_{k} \rightarrow \infty$ do we have $z\left(t_{k}\right) \rightarrow z^{c}$. Practically then, one could assume $z\left(t_{k}\right) \neq z^{c}$, which is true for any finite $k$ when $z\left(t_{-1}\right) \neq z^{c}$, and ignore the test completely. Also, if dual-mode receding horizon control is used, the test can be removed, since Proposition 2 is not used to prove asymptotic stability in that case. A dual-mode version will be provided in the next section.

If $J_{i}^{*}\left(z_{i}\left(t_{-1}\right), z_{-i}\left(t_{-1}\right), T\right)=0$ for any agent $i$, then it can be shown that $z_{i}\left(t_{-1}\right)=z_{i}^{c}$ and $z_{j}\left(t_{-1}\right)=z_{j}^{c}$, for each neighbor $j \in \mathcal{N}_{i}$, is the unique feasible solution, i.e., the local objective 
has been met. However, even if $J_{i}^{*}\left(z_{i}\left(t_{-1}\right), z_{-i}\left(t_{-1}\right), T\right)=0$, it may not remain zero for all $k \in$ $\mathbb{N}$. An example is where $i$ and all neighbors $j \in \mathcal{N}_{i}$ are initialized meeting their objective, but some $l \in \mathcal{N}_{j}$ has not met its objective. Thus, in the subsequent optimizations, $j$ will react to $l$, followed by $i$ reacting to $j$, since the coupling cost terms become nontrivial. Consequently, we can not guarantee that each distributed optimal value function $J_{i}^{*}\left(z_{i}\left(t_{k}\right), z_{-i}\left(t_{k}\right), T\right)$ will decrease with each receding horizon update. Instead, we show in the next section that the sum of the distributed optimal value functions is a Lyapunov function that does decrease at each update, enabling a proof that the distributed receding horizon control laws collectively meet the control objective.

\subsection{Stability Analysis}

We now proceed with analyzing the distributed receding horizon control laws. At any time $t_{k}, k \in \mathbb{N}$, the sum of the optimal distributed value functions is denoted as

$$
J_{\Sigma}^{*}\left(z\left(t_{k}\right), T\right)=\sum_{i=1}^{N_{a}} J_{i}^{*}\left(z_{i}\left(t_{k}\right), z_{-i}\left(t_{k}\right), T\right) .
$$

For stability of the distributed receding horizon control laws, we investigate $J_{\Sigma}^{*}\left(z\left(t_{k}\right), T\right)$ as a Lyapunov function.

Definition 9. Problem 2 is feasible at time $t_{k}$ if for every $i=1, \ldots, N_{a}$, there exists a control $u_{i}\left(\cdot ; z_{i}\left(t_{k}\right)\right):\left[t_{k}, t_{k}+T\right] \rightarrow \mathcal{U}$ such that all the constraints are satisfied and the value function $J_{i}\left(z_{i}\left(t_{k}\right), z_{-i}\left(t_{k}\right), u_{i}(\cdot), T\right)$ is bounded. Let $Z_{\Sigma} \subset \mathcal{Z}^{N_{a}}$ denote the set of initial states for which Problem 2 is feasible at initialization (time $t=t_{-1}$ ), as defined in Definition 7.

Lemma 1. Under Assumptions 1 and 3, $Z_{\Sigma}$ is a positively invariant set with respect to the closed-loop system (8) setting $u_{d i}^{*}\left(\cdot ; z_{i}\left(t_{k}\right)\right)=\hat{u}_{i}\left(\cdot ; z_{i}\left(t_{k}\right)\right)$ for every $i=1, \ldots, N_{a}$ and for $k \in \mathbb{N}$. Thus, feasibility at initialization implies subsequent feasibility.

The proof follows immediately from Definitions 7 and 8. Note that the assumed control $\hat{u}_{i}$ is exactly the feasible control trajectory used in Lemma 2 of [6] to show initial feasibility implies subsequent feasibility of the on-line optimization problem in the centralized case. Clearly, $z^{c}$ is in the set $Z_{\Sigma}$.

Remark 10. Since we will be exploring the closed-loop behavior for initial states that start in $Z_{\Sigma}$, we can immediately infer that any closed-loop state trajectory will remain bounded. Specifically, if an initial state can be driven to the compact terminal constraint set in finite time using bounded control $(\mathcal{U}$ is compact), then the optimal trajectory from that state will remain bounded. In the bounding argument for the proof of stability, we will make use of the notation

$$
\left\|z_{d i}^{*}\left(\tau ; t_{k}\right)-z_{i}^{c}\right\| \leq R, \quad \text { for any } \tau \in\left[t_{k}, t_{k}+T\right], k \in \mathbb{N}, \text { for all } i=1, \ldots, N_{a} .
$$

Moreover, let $U_{\max }>0$ be a positive scalar denoting the maximum-norm value over all feasible controls $u(t) \in \mathcal{U}$ at any time $t$. 
We make the following assumption.

Assumption 4. The optimal solution to Problem 2 exists and is numerically obtainable for any $z\left(t_{k}\right) \in Z_{\Sigma}$.

Given the additional assumption above, we have the following result.

Lemma 2. Under Assumptions 1, 3 and $4, Z_{\Sigma}$ is a positively invariant set with respect to the closed-loop system (8). Thus, if $z\left(t_{-1}\right) \in Z_{\Sigma}$, $z_{\text {dist }}^{*}(\tau) \in Z_{\Sigma}$ for all $\tau \geq t_{-1}$.

The next result says that the net objective of the distributed receding horizon control laws is consistent with the control objective.

Proposition 2. Under Assumptions 1, 3 and 4, for a given fixed horizon time $T>0$ and at any time $t_{k}, k \in \mathbb{N}$,

1. $J_{\Sigma}^{*}\left(z\left(t_{k}\right), T\right) \geq 0$ for any $z\left(t_{k}\right) \in Z_{\Sigma}$, and $J_{\Sigma}^{*}\left(z\left(t_{k}\right), T\right)=0$ if and only if $z\left(t_{k}\right)=z^{c}$,

2. $J_{\Sigma}^{*}\left(z\left(t_{k}\right), T\right)$ is continuous at $z\left(t_{k}\right)=z^{c}$.

Proof. The proposition is similar to Lemma A.1 in [5]. We prove item 1 here, while item 2 follows closely along the lines of the proof in the lemma.

1. The non-negativity of $J_{\Sigma}^{*}\left(z\left(t_{k}\right), T\right)$ follows directly from $L_{i}\left(z_{i}, \hat{z}_{-i}, u_{i}\right) \geq 0$ and $P_{i}>0$, for all $i=1, \ldots, N_{a}$. It remains therefore to prove that equality to zero is equivalent to $z\left(t_{k}\right)=z^{c}$.

$(\Rightarrow) J_{\Sigma}^{*}(z(t), T)=0$ implies that for each $i=1, \ldots, N_{a}$,

$$
\begin{gathered}
\gamma\left\|z_{d i}^{*}\left(t_{k}+T ; z_{i}\left(t_{k}\right)\right)-z_{i}^{c}\right\|_{P_{i}}^{2}=0 \quad \text { and } \\
\int_{t_{k}}^{t_{k}+T} L_{i}\left(z_{d i}^{*}\left(\tau ; z_{i}\left(t_{k}\right)\right), \hat{z}_{-i}\left(\tau ; \hat{z}_{-i}\left(t_{k}\right)\right), u_{d i}^{*}\left(\tau ; z_{i}\left(t_{k}\right)\right)\right) \mathrm{d} \tau=0 .
\end{gathered}
$$

Since the integrand is piece-wise continuous in $\tau$ on $\left[t_{k}, t_{k}+T\right]$ and nonnegative, we have that

$$
L_{i}\left(z_{d i}^{*}\left(\tau ; z_{i}\left(t_{k}\right)\right), \hat{z}_{-i}\left(\tau ; \hat{z}_{-i}\left(t_{k}\right)\right), u_{d i}^{*}\left(\tau ; z_{i}\left(t_{k}\right)\right)\right)=0, \quad \forall \tau \in\left[t_{k}, t_{k}+T\right],
$$

and for every $i=1, \ldots, N_{a}$. From Definition 5 , this implies $u_{d i}^{*}\left(\tau ; z_{i}\left(t_{k}\right)\right)=0$ and $\dot{q}_{d i}^{*}\left(\tau ; z_{i}\left(t_{k}\right)\right)=$ 0 , for all $\tau \in\left[t_{k}, t_{k}+T\right]$, meeting the control objective for the velocity for every $i$. Further, since the distributed optimal velocity is identically zero, $q_{d i}^{*}\left(\tau ; q_{i}\left(t_{k}\right)\right)=q_{i}\left(t_{k}\right)$, for all $\tau \in\left[t_{k}, t_{k}+T\right]$ and every $i$. Finally, from the distributed terminal costs, we have

$$
\gamma\left\|z_{i}\left(t_{k}\right)-z_{i}^{c}\right\|_{P_{i}}^{2}=0, \quad \text { for every } i=1, \ldots, N_{a} .
$$

Since every $P_{i}$ is positive definite and $\gamma$ is positive, $z_{i}\left(t_{k}\right)=z_{i}^{c}$ for every $i$, which is equivalent to $z\left(t_{k}\right)=z^{c}$. We must also guarantee that the resulting distributed optimal control and state are feasible. The constraints $z_{d i}^{*}\left(\tau ; z_{i}\left(t_{k}\right)\right) \in \mathcal{Z}$ and $u_{d i}^{*}\left(\tau ; z_{i}\left(t_{k}\right)\right) \in \mathcal{U}$ are trivial, since $z_{i}^{c}$ and 0 are in the interior of $\mathcal{Z}$ and $\mathcal{U}$, respectively, by Assumption 1. Also, $z_{i}^{c} \in \Omega_{i}\left(\varepsilon_{i}\right)$ so the terminal constraint is satisfied. Finally, since $z\left(t_{k}\right)=z^{c}$, by Definition $8, \hat{u}_{i}\left(\tau ; z_{i}\left(t_{k}\right)\right)=0$ for all $\tau \in\left[t_{k}, t_{k}+T\right]$ and every $i$, so the control comparison constraint is also satisfied. 
$(\Leftarrow)$ Given $z\left(t_{k}\right)=z^{c}$, by Definition 8 we have that for each $i=1, \ldots, N_{a}, \hat{u}_{i}\left(\tau ; z_{i}\left(t_{k}\right)\right)=0$ for all $\tau \in\left[t_{k}, t_{k}+T\right]$. Consequently, $\dot{\hat{q}}_{i}\left(\tau ; z_{i}\left(t_{k}\right)\right)=\dot{q}_{i}\left(t_{k}\right)=0$ and $\hat{q}_{i}\left(\tau ; z_{i}\left(t_{k}\right)\right)=q_{i}\left(t_{k}\right)=q_{i}^{c}$, for all $\tau \in\left[t_{k}, t_{k}+T\right]$, and every $i$. For any agent $i$, the local value function becomes

$$
\begin{aligned}
\int_{t_{k}}^{t_{k}+T} \gamma \sum_{j \in \mathcal{N}_{i}}\left\{\frac{\omega}{2}\left\|q_{i}\left(\tau ; z_{i}\left(t_{k}\right)\right)-q_{i}^{c}\right\|^{2}\right\} & +\gamma\left(\nu\left\|\dot{q}_{i}\left(\tau ; z_{i}\left(t_{k}\right)\right)\right\|^{2}+\mu\left\|u_{i}\left(\tau ; z_{i}\left(t_{k}\right)\right)\right\|^{2}\right) \mathrm{d} \tau \\
& +\gamma\left\|z_{i}\left(t_{k}+T ; z_{i}\left(t_{k}\right)\right)-z_{i}^{c}\right\|_{P_{i}}^{2},
\end{aligned}
$$

using $q_{i}^{c}+d_{i j}=q_{j}^{c}$, for each neighbor $j \in \mathcal{N}_{i}$. If $i$ is a core vehicle (i.e., $i$ is 1,2 or 3 ), we also have an addition term due to the tracking cost. In any case, given $z_{i}\left(t_{k}\right)=z_{i}^{c}$, the optimal and feasible control for each open-loop optimization problem is $u_{d i}^{*}\left(\tau ; z_{i}\left(t_{k}\right)\right)=0$ for all $\tau \in\left[t_{k}, t_{k}+T\right]$. Therefore, $\dot{q}_{d i}^{*}\left(\tau ; z_{i}\left(t_{k}\right)\right)=0$ and $q_{d i}^{*}\left(\tau ; q_{i}\left(t_{k}\right)\right)=q_{i}\left(t_{k}\right)=q_{i}^{c}$, for all $\tau \in\left[t_{k}, t_{k}+T\right]$. Since this holds for any $i$, every $J_{i}^{*}\left(z_{i}\left(t_{k}\right), z_{-i}\left(t_{k}\right), T\right)=0$ and so $J_{\Sigma}^{*}\left(z\left(t_{k}\right), T\right)=0$.

The condition if $z\left(t_{k}\right)=z^{c}$ then $\hat{u}_{i}\left(\tau ; z_{i}\left(t_{k}\right)\right)=0$ in Definition 8 was used in showing the equivalence of $J_{\Sigma}^{*}\left(z\left(t_{k}\right), T\right)=0$ and $z\left(t_{k}\right)=z^{c}$. For a dual-mode implementation, this equivalence is not required, eliminating the need for this condition in constructing any assumed control.

Our objective is to show the distributed receding horizon control law achieves the control objective for sufficiently small $\delta$. We begin with three lemmas that are used to bound the Lyapunov function candidate $J_{\Sigma}^{*}\left(z\left(t_{k}\right), T\right)$. The first lemma gives a bounding result on the decrease in $J_{\Sigma}^{*}(\cdot, T)$ from one update to the next.

Lemma 3. Under Assumptions 1, 3 and 4, for a given fixed horizon time $T>0$, there exists a positive constant $\xi>0$ such that

$$
J_{\Sigma}^{*}\left(z\left(t_{k+1}\right), T\right)-J_{\Sigma}^{*}\left(z\left(t_{k}\right), T\right) \leq-\int_{t_{k}}^{t_{k}+\delta} \sum_{i=1}^{N_{a}} L_{i}^{z}\left(z_{d i}^{*}\left(\tau ; z_{i}\left(t_{k}\right)\right), \hat{z}_{-i}\left(\tau ; z_{-i}\left(t_{k}\right)\right)\right) \mathrm{d} \tau+\delta^{2} \xi,
$$

for any $\delta \in(0, T]$ and for any $z\left(t_{k}\right) \in Z_{\Sigma}, k \in \mathbb{N}$.

Proof. The sum of the optimal distributed value functions for a given $z\left(t_{k}\right) \in Z_{\Sigma}$ is

$$
\begin{array}{r}
J_{\Sigma}^{*}\left(z\left(t_{k}\right), T\right)=\int_{t_{k}}^{t_{k}+T} \sum_{i=1}^{N_{a}} L_{i}\left(z_{d i}^{*}\left(\tau ; z_{i}\left(t_{k}\right)\right), \hat{z}_{-i}\left(\tau ; z_{-i}\left(t_{k}\right)\right), u_{d i}^{*}\left(\tau ; z_{i}\left(t_{k}\right)\right)\right) \mathrm{d} \tau \\
+\gamma\left\|z_{\text {dist }}^{*}\left(t_{k}+T ; z\left(t_{k}\right)\right)-z^{c}\right\|_{\widehat{P}}^{2} .
\end{array}
$$

Applying the optimal control for some $\delta \in(0, T]$ seconds, we are now at time $t_{k+1}=t_{k}+\delta$, with new state update $z\left(t_{k+1}\right)$. A feasible control for the optimal control problem of each agent over the new time interval $\tau \in\left[t_{k+1}, t_{k+1}+T\right]$ is $u_{i}\left(\cdot ; z_{i}\left(t_{k+1}\right)\right)=\hat{u}_{i}\left(\cdot ; z_{i}\left(t_{k+1}\right)\right)$, given 
in Definition 8. Thus, we have for any $i=1, \ldots, N_{a}$,

$$
\begin{array}{r}
J_{i}^{*}\left(z_{i}\left(t_{k+1}\right), z_{-i}\left(t_{k+1}\right), T\right)=\int_{t_{k+1}}^{t_{k+1}+T} L_{i}\left(z_{d i}^{*}\left(\tau ; z_{i}\left(t_{k+1}\right)\right), \hat{z}_{-i}\left(\tau ; z_{-i}\left(t_{k+1}\right)\right), u_{d i}^{*}\left(\tau ; z_{i}\left(t_{k+1}\right)\right)\right) \mathrm{d} \tau \\
+\gamma\left\|z_{d i}^{*}\left(t_{k+1}+T ; z_{i}\left(t_{k+1}\right)\right)-z_{i}^{c}\right\|_{P_{i}}^{2} \\
\leq \int_{t_{k+1}}^{t_{k+1}+T} L_{i}\left(\hat{z}_{i}\left(\tau ; z_{i}\left(t_{k+1}\right)\right), \hat{z}_{-i}\left(\tau ; z_{-i}\left(t_{k+1}\right)\right), \hat{u}_{i}\left(\tau ; z_{i}\left(t_{k+1}\right)\right)\right) \mathrm{d} \tau \\
+\gamma\left\|\hat{z}_{i}\left(t_{k+1}+T ; z_{i}\left(t_{k+1}\right)\right)-z_{i}^{c}\right\|_{P_{i} .}^{2} .
\end{array}
$$

Summing over $i$, we can upper bound $J_{\Sigma}^{*}\left(z\left(t_{k+1}\right), T\right)$, and comparing to $J_{\Sigma}^{*}\left(z\left(t_{k}\right), T\right)$ we have

$$
\begin{aligned}
& J_{\Sigma}^{*}\left(z\left(t_{k+1}\right), T\right)-J_{\Sigma}^{*}\left(z\left(t_{k}\right), T\right)+\int_{t_{k}}^{t_{k}+\delta} \sum_{i=1}^{N_{a}} L_{i}^{z}\left(z_{d i}^{*}\left(\tau ; z_{i}\left(t_{k}\right)\right), \hat{z}_{-i}\left(\tau ; z_{-i}\left(t_{k}\right)\right), u_{d i}^{*}\left(\tau ; z_{i}\left(t_{k}\right)\right)\right) \mathrm{d} \tau \\
& \leq \quad \int_{t_{k+1}}^{t_{k}+T} \sum_{i=1}^{N_{a}} L_{i}\left(\hat{z}_{i}\left(\tau ; z_{i}\left(t_{k+1}\right)\right), \hat{z}_{-i}\left(\tau ; z_{-i}\left(t_{k+1}\right)\right), \hat{u}_{i}\left(\tau ; z_{i}\left(t_{k+1}\right)\right)\right) \mathrm{d} \tau \\
&-\int_{t_{k+1}}^{t_{k}+T} \sum_{i=1}^{N_{a}} L_{i}\left(z_{d i}^{*}\left(\tau ; z_{i}\left(t_{k}\right)\right), \hat{z}_{-i}\left(\tau ; z_{-i}\left(t_{k}\right)\right), u_{d i}^{*}\left(\tau ; z_{i}\left(t_{k}\right)\right)\right) \mathrm{d} \tau \\
&+\int_{t_{k}+T}^{t_{k+1}+T} \sum_{i=1}^{N_{a}} L_{i}\left(\hat{z}_{i}\left(\tau ; z_{i}\left(t_{k+1}\right)\right), \hat{z}_{-i}\left(\tau ; z_{-i}\left(t_{k+1}\right)\right), \hat{u}_{i}\left(\tau ; z_{i}\left(t_{k+1}\right)\right)\right) \mathrm{d} \tau \\
&+\gamma \sum_{i=1}^{N_{a}}\left\|\hat{z}_{i}\left(t_{k+1}+T ; z_{i}\left(t_{k+1}\right)\right)-z_{i}^{c}\right\|_{P_{i}}^{2}-\gamma\left\|z_{\mathrm{dist}}^{*}\left(t_{k}+T ; z\left(t_{k}\right)\right)-z^{c}\right\|_{\widehat{P}}^{2}
\end{aligned}
$$

Using the notation $\hat{z}(\cdot ; z)=\left(\hat{z}_{1}\left(\cdot ; z_{1}\right), \ldots, \hat{z}_{N_{a}}\left(\cdot ; z_{N_{a}}\right)\right)$, we observe that

$$
\sum_{i=1}^{N_{a}}\left\|\hat{z}_{i}\left(t_{k+1}+T ; z_{i}\left(t_{k+1}\right)\right)-z_{i}^{c}\right\|_{P_{i}}^{2}=\left\|\hat{z}\left(t_{k+1}+T ; z\left(t_{k+1}\right)\right)-z^{c}\right\|_{\widehat{P}}^{2}
$$

and also that $\hat{z}\left(t_{k}+T ; z\left(t_{k+1}\right)\right)=z_{\text {dist }}^{*}\left(t_{k}+T ; z\left(t_{k}\right)\right)$. From the definition for each $\hat{u}_{i}\left(\tau ; z_{i}\left(t_{k+1}\right)\right)$ with $\tau \in\left[t_{k}+T, t_{k+1}+T\right]$ and using the notation in equation (10), we also have

$$
\sum_{i=1}^{N_{a}} L_{i}\left(\hat{z}_{i}\left(\tau ; z_{i}\left(t_{k+1}\right)\right), \hat{z}_{-i}\left(\tau ; z_{-i}\left(t_{k+1}\right)\right), \hat{u}_{i}\left(\tau ; z_{i}\left(t_{k+1}\right)\right)\right)=\gamma\left\|\hat{z}\left(\tau ; z\left(t_{k+1}\right)\right)-z^{c}\right\|_{\widetilde{Q}}^{2}
$$

where $\widetilde{Q}=Q+\mu \widehat{K}^{T} \widehat{K}$. Finally, from the properties of the feedback in Assumption 3 and the notation in equation (10), we have

$\left\|\hat{z}\left(t_{k+1}+T ; z\left(t_{k+1}\right)\right)-z^{c}\right\|_{\widehat{P}}^{2}-\left\|\hat{z}\left(t_{k}+T ; z\left(t_{k+1}\right)\right)-z^{c}\right\|_{\widehat{P}}^{2}=-\int_{t_{k}+T}^{t_{k+1}+T}\left\|\hat{z}\left(\tau ; z\left(t_{k+1}\right)\right)-z^{c}\right\|_{Q^{\prime}}^{2} \mathrm{~d} \tau$ 
where $Q^{\prime}=\widehat{Q}+\mu \widehat{K}^{T} \widehat{K}$ and $Q^{\prime} \geq \widetilde{Q}$. Thus, we can write

$$
\begin{aligned}
& \int_{t_{k}+T}^{t_{k+1}+T} \sum_{i=1}^{N_{a}} L_{i}\left(\hat{z}_{i}\left(\tau ; z_{i}\left(t_{k+1}\right)\right), \hat{z}_{-i}\left(\tau ; z_{-i}\left(t_{k+1}\right)\right), \hat{u}_{i}\left(\tau ; z_{i}\left(t_{k+1}\right)\right)\right) \mathrm{d} \tau \\
& +\gamma \sum_{i=1}^{N_{a}}\left\|\hat{z}_{i}\left(t_{k+1}+T ; z_{i}\left(t_{k+1}\right)\right)-z_{i}^{c}\right\|_{P_{i}}^{2}-\gamma\left\|z_{\text {dist }}^{*}\left(t_{k}+T ; z\left(t_{k}\right)\right)-z^{c}\right\|_{\widehat{P}}^{2} \\
& =-\gamma \int_{t_{k}+T}^{t_{k+1}+T}\left\|\hat{z}\left(\tau ; z\left(t_{k+1}\right)\right)-z^{c}\right\|_{Q^{\prime}-\widetilde{Q}}^{2} \mathrm{~d} \tau \leq 0 .
\end{aligned}
$$

Now, we have

$$
\begin{aligned}
J_{\Sigma}^{*}\left(z\left(t_{k+1}\right), T\right)-J_{\Sigma}^{*}\left(z\left(t_{k}\right), T\right)+\int_{t_{k}}^{t_{k}+\delta} \sum_{i=1}^{N_{a}} L_{i}^{z}\left(z_{d i}^{*}\left(\tau ; z_{i}\left(t_{k}\right)\right), \hat{z}_{-i}\left(\tau ; z_{-i}\left(t_{k}\right)\right), u_{d i}^{*}\left(\tau ; z_{i}\left(t_{k}\right)\right)\right) \mathrm{d} \tau \\
\leq \quad \int_{t_{k+1}}^{t_{k}+T} \sum_{i=1}^{N_{a}} L_{i}\left(\hat{z}_{i}\left(\tau ; z_{i}\left(t_{k+1}\right)\right), \hat{z}_{-i}\left(\tau ; z_{-i}\left(t_{k+1}\right)\right), \hat{u}_{i}\left(\tau ; z_{i}\left(t_{k+1}\right)\right)\right) \mathrm{d} \tau \\
\quad-\int_{t_{k+1}}^{t_{k}+T} \sum_{i=1}^{N_{a}} L_{i}\left(z_{d i}^{*}\left(\tau ; z_{i}\left(t_{k}\right)\right), \hat{z}_{-i}\left(\tau ; z_{-i}\left(t_{k}\right)\right), u_{d i}^{*}\left(\tau ; z_{i}\left(t_{k}\right)\right)\right) \mathrm{d} \tau .
\end{aligned}
$$

By definition, each $\hat{z}_{i}\left(\tau ; z_{i}\left(t_{k+1}\right)\right)=z_{d i}^{*}\left(\tau ; z_{i}\left(t_{k}\right)\right)$ and $\hat{u}_{i}\left(\tau ; z_{i}\left(t_{k+1}\right)\right)=u_{d i}^{*}\left(\tau ; z_{i}\left(t_{k}\right)\right)$, over the interval $\tau \in\left[t_{k+1}, t_{k}+T\right]$. Consequently, we have from Definition 5

$$
\begin{gathered}
\int_{t_{k+1}}^{t_{k}+T} \sum_{i=1}^{N_{a}} L_{i}\left(\hat{z}_{i}\left(\tau ; z_{i}\left(t_{k+1}\right)\right), \hat{z}_{-i}\left(\tau ; z_{-i}\left(t_{k+1}\right)\right), \hat{u}_{i}\left(\tau ; z_{i}\left(t_{k+1}\right)\right)\right) \mathrm{d} \tau \\
-\int_{t_{k+1}}^{t_{k}+T} \sum_{i=1}^{N_{a}} L_{i}\left(z_{d i}^{*}\left(\tau ; z_{i}\left(t_{k}\right)\right), \hat{z}_{-i}\left(\tau ; z_{-i}\left(t_{k}\right)\right), u_{d i}^{*}\left(\tau ; z_{i}\left(t_{k}\right)\right)\right) \mathrm{d} \tau \\
=\gamma \int_{t_{k+1}}^{t_{k}+T} \sum_{i=1}^{N_{a}} \sum_{j \in \mathcal{N}_{i}} \frac{\omega}{2}\left\{\left\|q_{d i}^{*}\left(\tau ; z_{i}\left(t_{k}\right)\right)-q_{d j}^{*}\left(\tau ; z_{j}\left(t_{k}\right)\right)+d_{i j}\right\|^{2}\right. \\
\quad \gamma \int_{t_{k+1}}^{t_{k}+T} \sum_{(i, j, l) \in \mathcal{E}_{c}} \frac{\omega}{27}\left\{\left\|q_{d i}^{*}\left(\tau ; z_{i}\left(t_{k}\right)\right)+q_{d j}^{*}\left(\tau ; z_{j}\left(t_{k}\right)\right)+q_{d l}^{*}\left(\tau ; z_{l}\left(t_{k}\right)\right)-3 q_{d}\right\|^{2}\right. \\
\left.-\left\|q_{d i}^{*}\left(\tau ; z_{i}\left(t_{k}\right)\right)+\hat{q}_{j}\left(\tau ; z_{j}\left(t_{k}\right)\right)+\hat{q}_{l}\left(\tau ; z_{l}\left(t_{k}\right)\right)-3 q_{d}\right\|^{2}\right\} \mathrm{d} \tau
\end{gathered}
$$

where $\mathcal{E}_{c}=\{(1,2,3),(3,1,2),(2,3,1)\}$. For the remainder of the proof, we are interested in finding an upper bound for the expression on the right hand side of the equality above. Consider the terms in the first integral

$$
\left\|q_{d i}^{*}\left(\tau ; z_{i}\left(t_{k}\right)\right)-q_{d j}^{*}\left(\tau ; z_{j}\left(t_{k}\right)\right)+d_{i j}\right\|^{2}-\left\|q_{d i}^{*}\left(\tau ; z_{i}\left(t_{k}\right)\right)-\hat{q}_{j}\left(\tau ; q_{j}\left(t_{k}\right)\right)+d_{i j}\right\|^{2},
$$


over the interval $\tau \in\left[t_{k+1}, t_{k}+T\right]$. The first term can be bounded using the triangle inequality as

$$
\begin{aligned}
& \left\|q_{d i}^{*}\left(\tau ; z_{i}\left(t_{k}\right)\right)-q_{d j}^{*}\left(\tau ; z_{j}\left(t_{k}\right)\right)+d_{i j}\right\|^{2} \\
& \quad=\left\|q_{d i}^{*}\left(\tau ; z_{i}\left(t_{k}\right)\right)-q_{d j}^{*}\left(\tau ; z_{j}\left(t_{k}\right)\right) \pm \hat{q}_{j}\left(\tau ; q_{j}\left(t_{k}\right)\right)+d_{i j}\right\|^{2} \\
& \quad \begin{array}{l}
\left\|q_{d i}^{*}\left(\tau ; z_{i}\left(t_{k}\right)\right)-\hat{q}_{j}\left(\tau ; q_{j}\left(t_{k}\right)\right)+d_{i j}\right\|^{2}+\left\|\hat{q}_{j}\left(\tau ; q_{j}\left(t_{k}\right)\right)-q_{d j}^{*}\left(\tau ; z_{j}\left(t_{k}\right)\right)\right\|^{2} \\
\quad \\
\quad+2\left\|q_{d i}^{*}\left(\tau ; z_{i}\left(t_{k}\right)\right)-\hat{q}_{j}\left(\tau ; q_{j}\left(t_{k}\right)\right)+d_{i j}\right\| \cdot\left\|\hat{q}_{j}\left(\tau ; q_{j}\left(t_{k}\right)\right)-q_{d j}^{*}\left(\tau ; z_{j}\left(t_{k}\right)\right)\right\| .
\end{array}
\end{aligned}
$$

With this upper bound, we have a cancellation with the negative term $\| q_{d i}^{*}\left(\tau ; z_{i}\left(t_{k}\right)\right)-$ $\hat{q}_{j}\left(\tau ; q_{j}\left(t_{k}\right)\right)+d_{i j} \|^{2}$ in the integral. Now, considering the terms in the second integral, particularly the first term, we can use that same bounding argument to get

$$
\begin{aligned}
& \left\|q_{d i}^{*}\left(\tau ; z_{i}\left(t_{k}\right)\right)+q_{d j}^{*}\left(\tau ; z_{j}\left(t_{k}\right)\right)+q_{d l}^{*}\left(\tau ; z_{l}\left(t_{k}\right)\right)-3 q_{d}\right\|^{2} \\
& =\left\|q_{d i}^{*}\left(\tau ; z_{i}\left(t_{k}\right)\right)+q_{d j}^{*}\left(\tau ; z_{j}\left(t_{k}\right)\right)+q_{d l}^{*}\left(\tau ; z_{l}\left(t_{k}\right)\right) \pm \hat{q}_{j}\left(\tau ; z_{j}\left(t_{k}\right)\right) \pm \hat{q}_{l}\left(\tau ; z_{l}\left(t_{k}\right)\right)-3 q_{d}\right\|^{2} \\
& \leq\left\|q_{d i}^{*}\left(\tau ; z_{i}\left(t_{k}\right)\right)+\hat{q}_{j}\left(\tau ; z_{j}\left(t_{k}\right)\right)+\hat{q}_{l}\left(\tau ; z_{l}\left(t_{k}\right)\right)-3 q_{d}\right\|^{2} \\
& \quad+2\left\|q_{d i}^{*}\left(\tau ; z_{i}\left(t_{k}\right)\right)+\hat{q}_{j}\left(\tau ; z_{j}\left(t_{k}\right)\right)+\hat{q}_{l}\left(\tau ; z_{l}\left(t_{k}\right)\right)-3 q_{d}\right\| \\
& \quad \cdot\left\|q_{d j}^{*}\left(\tau ; z_{j}\left(t_{k}\right)\right)-\hat{q}_{j}\left(\tau ; z_{j}\left(t_{k}\right)\right)+q_{d l}^{*}\left(\tau ; z_{l}\left(t_{k}\right)\right)-\hat{q}_{l}\left(\tau ; z_{l}\left(t_{k}\right)\right)\right\| \\
& \quad+\left\|q_{d j}^{*}\left(\tau ; z_{j}\left(t_{k}\right)\right)-\hat{q}_{j}\left(\tau ; z_{j}\left(t_{k}\right)\right)+q_{d l}^{*}\left(\tau ; z_{l}\left(t_{k}\right)\right)-\hat{q}_{l}\left(\tau ; z_{l}\left(t_{k}\right)\right)\right\|^{2} .
\end{aligned}
$$

Using the triangle inequality again we bound the term

$$
\begin{aligned}
& \left\|q_{d j}^{*}\left(\tau ; z_{j}\left(t_{k}\right)\right)-\hat{q}_{j}\left(\tau ; z_{j}\left(t_{k}\right)\right)+q_{d l}^{*}\left(\tau ; z_{l}\left(t_{k}\right)\right)-\hat{q}_{l}\left(\tau ; z_{l}\left(t_{k}\right)\right)\right\| \\
& \quad \leq\left\|q_{d j}^{*}\left(\tau ; z_{j}\left(t_{k}\right)\right)-\hat{q}_{j}\left(\tau ; z_{j}\left(t_{k}\right)\right)\right\|+\left\|q_{d l}^{*}\left(\tau ; z_{l}\left(t_{k}\right)\right)-\hat{q}_{l}\left(\tau ; z_{l}\left(t_{k}\right)\right)\right\| .
\end{aligned}
$$

Substitution back into the integral equations, after cancellations, yields an upper bound given as

$$
\begin{aligned}
\gamma \int_{t_{k+1}}^{t_{k}+T} \sum_{i=1}^{N_{a}} \sum_{j \in \mathcal{N}_{i}} \frac{\omega}{2}\left\{2\left\|q_{d i}^{*}\left(\tau ; z_{i}\left(t_{k}\right)\right)-\hat{q}_{j}\left(\tau ; q_{j}\left(t_{k}\right)\right)+d_{i j}\right\| \cdot\left\|\hat{q}_{j}\left(\tau ; q_{j}\left(t_{k}\right)\right)-q_{d j}^{*}\left(\tau ; z_{j}\left(t_{k}\right)\right)\right\|\right. \\
\left.+\left\|\hat{q}_{j}\left(\tau ; q_{j}\left(t_{k}\right)\right)-q_{d j}^{*}\left(\tau ; z_{j}\left(t_{k}\right)\right)\right\|^{2}\right\} \mathrm{d} \tau \\
+\gamma \int_{t_{k+1}}^{t_{k}+T} \sum_{(i, j, l) \in \mathcal{E}_{c}} \frac{\omega}{27}\left\{2\left\|q_{d i}^{*}\left(\tau ; z_{i}\left(t_{k}\right)\right)+\hat{q}_{j}\left(\tau ; z_{j}\left(t_{k}\right)\right)+\hat{q}_{l}\left(\tau ; z_{l}\left(t_{k}\right)\right)-3 q_{d}\right\|\right. \\
\cdot\left(\left\|q_{d j}^{*}\left(\tau ; z_{j}\left(t_{k}\right)\right)-\hat{q}_{j}\left(\tau ; z_{j}\left(t_{k}\right)\right)\right\|+\left\|q_{d l}^{*}\left(\tau ; z_{l}\left(t_{k}\right)\right)-\hat{q}_{l}\left(\tau ; z_{l}\left(t_{k}\right)\right)\right\|\right) \\
\left.+\left(\left\|q_{d j}^{*}\left(\tau ; z_{j}\left(t_{k}\right)\right)-\hat{q}_{j}\left(\tau ; z_{j}\left(t_{k}\right)\right)\right\|+\left\|q_{d l}^{*}\left(\tau ; z_{l}\left(t_{k}\right)\right)-\hat{q}_{l}\left(\tau ; z_{l}\left(t_{k}\right)\right)\right\|\right)^{2}\right\} \mathrm{d} \tau .
\end{aligned}
$$

Since each term in the integral is non-negative, we can upper bound this expression by extending the interval of integration from $\left[t_{k+1}, t_{k}+T\right]$ to $\left[t_{k}, t_{k}+T\right]$. The reason for doing this is that for any $j=1, \ldots, N_{a}, z_{d j}^{*}\left(t_{k} ; z_{j}\left(t_{k}\right)\right)=\hat{z}_{j}\left(t_{k} ; z_{j}\left(t_{k}\right)\right)$, thereby matching the initial 
conditions for the two trajectories we are comparing. Now, for any $j$, we can bound the term

$$
\begin{aligned}
\left\|q_{d j}^{*}\left(\tau ; z_{j}\left(t_{k}\right)\right)-\hat{q}_{j}\left(\tau ; z_{j}\left(t_{k}\right)\right)\right\| & \leq \int_{t_{k}}^{\tau}\left\|\dot{q}_{d j}^{*}\left(s ; z_{j}\left(t_{k}\right)\right)-\dot{\hat{q}}_{j}\left(s ; z_{j}\left(t_{k}\right)\right)\right\| \mathrm{d} s \\
& \leq \int_{t_{k}}^{\tau} \int_{t_{k}}^{s}\left\|u_{d j}^{*}\left(\eta ; z_{j}\left(t_{k}\right)\right)-\hat{u}_{j}\left(\eta ; z_{j}\left(t_{k}\right)\right)\right\| \mathrm{d} \eta \mathrm{d} s \\
& \leq \int_{t_{k}}^{\tau} \int_{t_{k}}^{s} \kappa \delta^{2} \mathrm{~d} \eta \mathrm{d} s=\frac{1}{2} \kappa \delta^{2}\left(\tau-t_{k}\right)^{2} .
\end{aligned}
$$

From equation (11), and using the triangle inequality and $q_{i}^{c}+d_{i j}=q_{j}^{c}$, we can also bound the term

$$
\left\|q_{d i}^{*}\left(\tau ; z_{i}\left(t_{k}\right)\right)-\hat{q}_{j}\left(\tau ; q_{j}\left(t_{k}\right)\right)+d_{i j}\right\| \leq\left\|q_{d i}^{*}\left(\tau ; z_{i}\left(t_{k}\right)\right)-q_{i}^{c}\right\|+\left\|\hat{q}_{j}\left(\tau ; q_{j}\left(t_{k}\right)\right)-q_{j}^{c}\right\| \leq 2 R .
$$

Similarly, using $q_{1}^{c}+q_{2}^{c}+q_{3}^{c}=3 q_{d}$, we have

$$
\begin{aligned}
& \left\|q_{d i}^{*}\left(\tau ; z_{i}\left(t_{k}\right)\right)+\hat{q}_{j}\left(\tau ; z_{j}\left(t_{k}\right)\right)+\hat{q}_{l}\left(\tau ; z_{l}\left(t_{k}\right)\right)-3 q_{d}\right\| \\
& \quad \leq\left\|q_{d i}^{*}\left(\tau ; z_{i}\left(t_{k}\right)\right)-q_{i}^{c}\right\|+\left\|\hat{q}_{j}\left(\tau ; q_{j}\left(t_{k}\right)\right)-q_{j}^{c}\right\|+\left\|\hat{q}_{l}\left(\tau ; q_{l}\left(t_{k}\right)\right)-q_{l}^{c}\right\| \leq 3 R .
\end{aligned}
$$

Note that $\sum_{i=1}^{N_{a}} \sum_{j \in \mathcal{N}_{i}} 1 / 2=M$, where $M$ is the number of relative vectors used to define the formation objective in Section 2. Substitution in the integral equations yields the upper bound

$$
\begin{aligned}
\gamma \int_{t_{k}}^{t_{k}+T}[ & \sum_{i=1}^{N_{a}} \sum_{j \in \mathcal{N}_{i}} \frac{\omega}{2}\left\{2 R \kappa \delta^{2}\left(\tau-t_{k}\right)^{2}+\kappa^{2} \delta^{4}\left(\tau-t_{k}\right)^{4} / 4\right\} \\
& \left.+\sum_{(i, j, l) \in \mathcal{E}_{c}} \frac{\omega}{27}\left\{6 R \kappa \delta^{2}\left(\tau-t_{k}\right)^{2}+\kappa^{2} \delta^{4}\left(\tau-t_{k}\right)^{4}\right\}\right] \mathrm{d} \tau \\
= & \gamma \omega \int_{t_{k}}^{t_{k}+T}\left[(6 M+2) R \kappa \delta^{2}\left(\tau-t_{k}\right)^{2} / 3+(5 M / 4+5 / 9) \kappa^{2} \delta^{4}\left(\tau-t_{k}\right)^{4} / 5\right] \mathrm{d} \tau \\
\leq & \delta^{2} \gamma \omega \kappa T^{3}(2 M+2 / 3)\left[3 R+\kappa \delta^{2} T^{2}\right] .
\end{aligned}
$$

For the last term in the brackets to be a constant independent of $\delta$, we can bound the expression by setting $\delta=T$ inside the brackets, since $\delta \in(0, T]$. Finally, we have

$$
\begin{aligned}
J_{\Sigma}^{*}\left(z\left(t_{k+1}\right), T\right)- & J_{\Sigma}^{*}\left(z\left(t_{k}\right), T\right) \\
& \leq-\int_{t_{k}}^{t_{k}+\delta} \sum_{i=1}^{N_{a}} L_{i}^{z}\left(z_{d i}^{*}\left(\tau ; z_{i}\left(t_{k}\right)\right), \hat{z}_{-i}\left(\tau ; z_{-i}\left(t_{k}\right)\right), u_{d i}^{*}\left(\tau ; z_{i}\left(t_{k}\right)\right)\right) \mathrm{d} \tau+\delta^{2} \xi \\
& \leq-\int_{t_{k}}^{t_{k}+\delta} \sum_{i=1}^{N_{a}} L_{i}^{z}\left(z_{d i}^{*}\left(\tau ; z_{i}\left(t_{k}\right)\right), \hat{z}_{-i}\left(\tau ; z_{-i}\left(t_{k}\right)\right)\right) \mathrm{d} \tau+\delta^{2} \xi,
\end{aligned}
$$


where

$$
\xi=\gamma \omega \kappa T^{3}(2 M+2 / 3)\left[3 R+\kappa T^{4}\right] .
$$

This completes the proof.

Ultimately, we want to show that $J_{\Sigma}^{*}(\cdot, T)$ decreases from one update to the next along the actual closed-loop trajectories. The next two lemmas show that, for sufficiently small $\delta$, the bounding expression above can be bounded by a negative-definite function of the closed-loop trajectories.

Lemma 4. Under Assumptions 1, 3 and 4, for any $z\left(t_{k}\right) \in Z_{\Sigma}, k \in \mathbb{N}$, such that at least one agent $i$ satisfies $z_{i}\left(t_{k}\right) \neq z_{i}^{c}$, and for any positive constant $\xi$, there exists a $\delta\left(z\left(t_{k}\right)\right)>0$ such that

$$
\int_{t_{k}}^{t_{k}+\delta} \sum_{i=1}^{N_{a}} L_{i}^{z}\left(z_{d i}^{*}\left(\tau ; z_{i}\left(t_{k}\right)\right), \hat{z}_{-i}\left(\tau ; z_{-i}\left(t_{k}\right)\right)\right) \mathrm{d} \tau-\delta^{2} \xi \geq \int_{t_{k}}^{t_{k}+\delta}\left\|z_{\text {dist }}^{*}\left(\tau ; z\left(t_{k}\right)\right)-z^{c}\right\|_{Q}^{2} \mathrm{~d} \tau,
$$

for any $\delta \in\left(0, \delta\left(z\left(t_{k}\right)\right)\right]$. If $z\left(t_{k}\right)=z^{c}$, then the equation above holds with $\delta\left(z\left(t_{k}\right)\right)=0$.

Proof. At time $\tau=t_{k}, z_{d i}^{*}\left(t_{k} ; z_{i}\left(t_{k}\right)\right)=z_{i}\left(t_{k}\right)$ and $\hat{z}_{-i}\left(\tau ; z_{-i}\left(t_{k}\right)\right)=z_{-i}\left(t_{k}\right)$, and so

$$
\sum_{i=1}^{N_{a}} L_{i}^{z}\left(z_{d i}^{*}\left(t_{k} ; z_{i}\left(t_{k}\right)\right), \hat{z}_{-i}\left(t_{k} ; z_{-i}\left(t_{k}\right)\right)\right)=\sum_{i=1}^{N_{a}} L_{i}^{z}\left(z_{i}\left(t_{k}\right), z_{-i}\left(t_{k}\right)\right)=\gamma\left\|z\left(t_{k}\right)-z^{c}\right\|_{Q}^{2},
$$

where the last equality is from Definition 5. Since $Q>0, \gamma>1$, and at least one agent $i$ satisfies $z_{i}\left(t_{k}\right) \neq z_{i}^{c}$, we have that $z\left(t_{k}\right) \neq z^{c}$ and

$$
\sum_{i=1}^{N_{a}} L_{i}^{z}\left(z_{d i}^{*}\left(t_{k} ; z_{i}\left(t_{k}\right)\right), \hat{z}_{-i}\left(t_{k} ; z_{-i}\left(t_{k}\right)\right)\right)>\left\|z\left(t_{k}\right)-z^{c}\right\|_{Q}^{2}>0
$$

Equivalently, we have

$$
\sum_{i=1}^{N_{a}} L_{i}^{z}\left(z_{d i}^{*}\left(t_{k} ; z_{i}\left(t_{k}\right)\right), \hat{z}_{-i}\left(t_{k} ; z_{-i}\left(t_{k}\right)\right)\right)>\left\|z_{\text {dist }}^{*}\left(t_{k} ; z\left(t_{k}\right)\right)-z^{c}\right\|_{Q}^{2}
$$

Under the assumptions, $\left.z_{d i}^{*}\left(\tau ; z_{i}\left(t_{k}\right)\right), z_{-i}(s)\right)$ and $\hat{z}_{-i}\left(\tau ; z_{-i}\left(t_{k}\right)\right)$ are absolutely continuous in $\tau$, for any $i=1, \ldots, N_{a}$. Any quadratic function of $z_{d i}^{*}$ and $\hat{z}_{-i}$, including each $L_{i}^{z}$, is therefore absolutely continuous in $\tau$. Thus, for any given $\xi>0$, we can choose a $\delta\left(z\left(t_{k}\right)\right)>0$ such that for any $\tau \in\left[t_{k}, t_{k}+\delta\left(z\left(t_{k}\right)\right)\right)$,

$$
\sum_{i=1}^{N_{a}} L_{i}^{z}\left(z_{d i}^{*}\left(\tau ; z_{i}\left(t_{k}\right)\right), \hat{z}_{-i}\left(\tau ; z_{-i}\left(t_{k}\right)\right)\right)>2\left(\tau-t_{k}\right) \xi+\left\|z_{\text {dist }}^{*}\left(\tau ; z\left(t_{k}\right)\right)-z^{c}\right\|_{Q}^{2}
$$


and equality holds when $\tau=t_{k}+\delta\left(z\left(t_{k}\right)\right)$. That is, $t_{k}+\delta\left(z\left(t_{k}\right)\right)$ is the first time at which the two sides of the inequality are equal. Choosing such a $\delta\left(z\left(t_{k}\right)\right)>0$ is possible even if each $L_{i}^{z}$ is a decreasing function of $\tau$ and $\left\|z_{\text {dist }}^{*}\left(\tau ; z\left(t_{k}\right)\right)-z^{c}\right\|_{Q}^{2}$ is an increasing function of $\tau$, because of the initial margin in equation (13) and also because the functions have bounded rate. The latter statement follows from the functions being quadratic, therefore the gradients are linear, and from the bounds on the state and control trajectories, as stated in Remark 10. By integrating both sides in $\tau$ over the interval $\left[t_{k}, t_{k}+\delta\left(z\left(t_{k}\right)\right)\right]$, the inequality still clearly holds. However, a larger value of $\delta\left(z\left(t_{k}\right)\right)$ can be obtained, since we are interested in comparing the integral equations in the first place, rather than comparing the expressions over all values of $\tau \in\left[t_{k}, t_{k}+\delta\left(z\left(t_{k}\right)\right)\right]$. Again, even assuming in the worst case that each $L_{i}^{z}$ is a decreasing function of $\tau$, while assuming $\left\|z_{\text {dist }}^{*}\left(\tau ; z\left(t_{k}\right)\right)-z^{c}\right\|_{Q}^{2}$ is an increasing function of $\tau$, we still have that for any given $\xi>0$, there exists a $\delta\left(z\left(t_{k}\right)\right)>0$ such that

$$
\begin{aligned}
\int_{t_{k}}^{t_{k}+\delta\left(z\left(t_{k}\right)\right)} \sum_{i=1}^{N_{a}} L_{i}^{z}\left(z_{d i}^{*}\left(\tau ; z_{i}\left(t_{k}\right)\right), \hat{z}_{-i}\left(\tau ; z_{-i}\left(t_{k}\right)\right)\right) \mathrm{d} \tau \\
=\int_{t_{k}}^{t_{k}+\delta\left(z\left(t_{k}\right)\right)} 2\left(\tau-t_{k}\right) \xi+\left\|z_{\text {dist }}^{*}\left(\tau ; z\left(t_{k}\right)\right)-z^{c}\right\|_{Q}^{2} \mathrm{~d} \tau \\
=\delta\left(z\left(t_{k}\right)\right)^{2} \xi+\int_{t_{k}}^{t_{k}+\delta\left(z\left(t_{k}\right)\right)}\left\|z_{\text {dist }}^{*}\left(\tau ; z\left(t_{k}\right)\right)-z^{c}\right\|_{Q}^{2} \mathrm{~d} \tau
\end{aligned}
$$

Finally, we have

$$
\int_{t_{k}}^{t_{k}+\delta} \sum_{i=1}^{N_{a}} L_{i}^{z}\left(z_{d i}^{*}\left(\tau ; z_{i}\left(t_{k}\right)\right), \hat{z}_{-i}\left(\tau ; z_{-i}\left(t_{k}\right)\right)\right) \mathrm{d} \tau \geq \delta^{2} \xi+\int_{t_{k}}^{t_{k}+\delta}\left\|z_{\mathrm{dist}}^{*}\left(\tau ; z\left(t_{k}\right)\right)-z^{c}\right\|_{Q}^{2} \mathrm{~d} \tau,
$$

for any $\delta>0$ no bigger than $\delta\left(z\left(t_{k}\right)\right)$, i.e., for any $\delta \in\left(0, \delta\left(z\left(t_{k}\right)\right)\right]$. If $z\left(t_{k}\right)=z^{c}$, then both integrands are identically zero (see proof of Proposition 2). As a result, we immediately have $\delta\left(z\left(t_{k}\right)\right)=0$.

Remark 11. For a given value of $\gamma>1$, as $\left\|z\left(t_{k}\right)-z^{c}\right\|$ decreases, so does the margin in equation (13). Consequently, as the states approach the control objective, the value of $\delta\left(z\left(t_{k}\right)\right)$ that satisfies the integral equality in equation (14) decreases. In fact, as $z\left(t_{k}\right) \rightarrow z^{c}$, the equation requires that $\delta\left(z\left(t_{k}\right)\right) \rightarrow 0$. This corresponds to an increasingly strict constraint on the deviation of the optimized control from the assumed control as $\left\|z\left(t_{k}\right)-z^{c}\right\|$ decreases. It also requires that communication of control trajectories must happen at an increasing rate, and with infinite bandwidth in the limit. Later, we will construct an update time that is sufficiently small to guarantee that all agents have reached their terminal constraint sets via the distributed receding horizon control, making it safe to henceforth apply the decoupled linear feedbacks (dual-mode). The sufficiently small value for the update period then serves as an upper bound on how small the value of $\delta$ must be for dual-mode distributed receding horizon control. 
The lemma above provides a test on the update period that later is used to guarantee distributed receding horizon control stability. It would more be useful to have an analytic expression for the test. Such an expression is difficult to obtain, since the trajectories in the integrals in equation (14) are implicity defined and therefore hard to analyze. However, by making an assumption that approximates and simplifies the functions in the integrals, we are able to obtain an analytic bound on the update period.

Assumption 5. The interval of integration $\left[t_{k}, t_{k}+\delta\right]$ for the expressions in equation (14) is sufficiently small that first-order Taylor series approximations of the integrands is a valid approximation for any $z\left(t_{k}\right) \in Z_{\Sigma}$. Specifically, we take

$$
\begin{aligned}
& \sum_{i=1}^{N_{a}} L_{i}^{z}\left(z_{d i}^{*}\left(\tau ; z_{i}\left(t_{k}\right)\right), \hat{z}_{-i}\left(\tau ; z_{-i}\left(t_{k}\right)\right)\right) \\
& \approx \gamma\left\|z\left(t_{k}\right)-z^{c}\right\|_{Q}^{2}+\left(\tau-t_{k}\right) \sum_{i=1}^{N_{a}}\left\{\nabla_{z_{i}} L_{i}^{z}\left(z_{i}\left(t_{k}\right), z_{-i}\left(t_{k}\right)\right)^{T}\left(A_{i} z_{i}\left(t_{k}\right)+B_{i} u_{d i}^{*}\left(t_{k} ; z_{i}\left(t_{k}\right)\right)\right)\right. \\
& \left.+\Sigma_{j \in \mathcal{N}_{i}} \nabla_{z_{j}} L_{i}^{z}\left(z_{i}\left(t_{k}\right), z_{-i}\left(t_{k}\right)\right)^{T}\left(A_{j} z_{j}\left(t_{k}\right)+B_{j} \hat{u}_{j}\left(t_{k} ; z_{j}\left(t_{k}\right)\right)\right)\right\}
\end{aligned}
$$

and

$\left\|z_{\text {dist }}^{*}\left(\tau ; z\left(t_{k}\right)\right)-z^{c}\right\|_{Q}^{2} \approx\left\|z\left(t_{k}\right)-z^{c}\right\|_{Q}^{2}+\left(\tau-t_{k}\right) 2\left(z\left(t_{k}\right)-z^{c}\right)^{T} Q\left(A z\left(t_{k}\right)+B u_{\text {dist }}^{*}\left(t_{k} ; z\left(t_{k}\right)\right)\right)$, and ignore terms that are $\mathrm{O}\left(\left(\tau-t_{k}\right)^{2}\right)$ and higher-order.

Lemma 5. Under Assumptions 1 and 3-5, for any $z\left(t_{k}\right) \in Z_{\Sigma}, k \in \mathbb{N}$, the margin in Lemma 4 is attained with

$$
\delta\left(z\left(t_{k}\right)\right)=\frac{(\gamma-1)\left\|z\left(t_{k}\right)-z^{c}\right\|_{Q}^{2}}{\xi+(\gamma-1) \lambda_{\max }(Q)\left(R^{2}+U_{\max }^{2}\right)},
$$

given the state and control bounds $R$ and $U_{\max }$, respectively.

Proof. From equation (14), for a given $z\left(t_{k}\right)$, we want to choose a $\delta$ such that

$$
\int_{t_{k}}^{t_{k}+\delta} \sum_{i=1}^{N_{a}} L_{i}^{z}\left(z_{d i}^{*}\left(\tau ; z_{i}\left(t_{k}\right)\right), \hat{z}_{-i}\left(\tau ; z_{-i}\left(t_{k}\right)\right)\right)-\left\|z_{\text {dist }}^{*}\left(\tau ; z\left(t_{k}\right)\right)-z^{c}\right\|_{Q}^{2} \mathrm{~d} \tau-\delta^{2} \xi \geq 0,
$$

with equality when $\delta=\delta\left(z\left(t_{k}\right)\right)$. Substitution of the Taylor series expression from Assumption 5 into the integrals results in

$$
\delta(\xi-C) \leq(\gamma-1)\left\|z\left(t_{k}\right)-z^{c}\right\|_{Q}^{2},
$$


where $C$ combines the first order terms in the expansions, given as

$$
\begin{aligned}
C=\left[\sum _ { i = 1 } ^ { N _ { a } } \left\{\frac{1}{2} \nabla_{q_{i}}\right.\right. & L_{i}^{z}\left(z_{i}\left(t_{k}\right), z_{-i}\left(t_{k}\right)\right)^{T} \dot{q}_{i}\left(t_{k}\right)+\gamma \nu \dot{q}_{i}\left(t_{k}\right)^{T} u_{d i}^{*}\left(t_{k} ; z_{i}\left(t_{k}\right)\right) \\
& \left.+\Sigma_{j \in \mathcal{N}_{i}} \frac{1}{2} \nabla_{q_{j}} L_{i}^{z}\left(z_{i}\left(t_{k}\right), z_{-i}\left(t_{k}\right)\right)^{T} \dot{q}_{j}\left(t_{k}\right)\right\} \\
& \left.-\left\{\omega\left(q\left(t_{k}\right)-q^{c}\right)^{T} G^{T} G \dot{q}\left(t_{k}\right)+\nu \dot{q}\left(t_{k}\right)^{T} u_{\text {dist }}^{*}\left(t_{k} ; z\left(t_{k}\right)\right)\right\}\right]
\end{aligned}
$$

Note that the dependence on $\hat{u}_{i}$ disappears since each $L_{i}$ is not a function of $\dot{q}_{j}$ for any neighbor $j \in \mathcal{N}_{i}$. Since all $\nabla L_{i}$ inner-product terms are consistent with the state $z\left(t_{k}\right)$, we can combine the sum to be

$$
\begin{array}{r}
\sum_{i=1}^{N_{a}}\left\{\frac{1}{2} \nabla_{q_{i}} L_{i}^{z}\left(z_{i}\left(t_{k}\right), z_{-i}\left(t_{k}\right)\right)^{T} \dot{q}_{i}\left(t_{k}\right)+\Sigma_{j \in \mathcal{N}_{i}} \frac{1}{2}\right. \\
\left.\nabla_{q_{j}} L_{i}^{z}\left(z_{i}\left(t_{k}\right), z_{-i}\left(t_{k}\right)\right)^{T} \dot{q}_{j}\left(t_{k}\right)\right\} \\
=\gamma \omega\left(q\left(t_{k}\right)-q^{c}\right)^{T} G^{T} G \dot{q}\left(t_{k}\right) .
\end{array}
$$

Consequently, we can simplify $C$ as

$$
\begin{aligned}
C & =(\gamma-1)\left[\omega\left(q\left(t_{k}\right)-q^{c}\right)^{T} G^{T} G \dot{q}\left(t_{k}\right)+\nu \dot{q}\left(t_{k}\right)^{T} u_{\text {dist }}^{*}\left(t_{k} ; z\left(t_{k}\right)\right)\right] \\
& =(\gamma-1)\left(z\left(t_{k}\right)-z^{c}\right)^{T} Q \dot{z}_{\text {dist }}^{*}\left(t_{k} ; z\left(t_{k}\right)\right) .
\end{aligned}
$$

Since $C$ is the inner-product of (in general) different vectors, it could be positive or negative. In equation (15), $(\gamma-1)>0$ is given and $\xi>0$ is given and we are looking for the largest $\delta$ such that the inequality holds. Note that if $C \geq \xi$, the inequality holds for any positive $\delta$. In the worst-case, the constant $C$ will be a negative number, removing more of the margin for $\delta$. More precisely, we first observe that for any two vectors $x$ and $y$,

$$
x^{T} y \geq-\max \left\{\|x\|^{2},\|y\|^{2}\right\} .
$$

From this, and using the bound assumptions in equation (11), namely

$$
\left\|z\left(t_{k}\right)-z^{c}\right\|^{2} \leq R^{2}, \quad \text { and } \quad\left\|\dot{z}_{\text {dist }}^{*}\left(t_{k} ; z\left(t_{k}\right)\right)\right\|^{2} \leq R^{2}+U_{\max }^{2},
$$

we have

$$
\left(Q^{1 / 2}\left(z\left(t_{k}\right)-z^{c}\right)\right)^{T}\left(Q^{1 / 2} \dot{z}_{\text {dist }}^{*}\left(t_{k} ; z\left(t_{k}\right)\right)\right) \geq-\lambda_{\max }(Q)\left(R^{2}+U_{\max }^{2}\right) .
$$

So, in the worst case, we have that $\delta$ must satisfy

$$
\delta\left[\xi+(\gamma-1) \lambda_{\max }(Q)\left(R^{2}+U_{\max }^{2}\right)\right] \leq(\gamma-1)\left\|z\left(t_{k}\right)-z^{c}\right\|_{Q}^{2},
$$

or, equivalently,

$$
\delta \leq \frac{(\gamma-1)\left\|z\left(t_{k}\right)-z^{c}\right\|_{Q}^{2}}{\xi+(\gamma-1) \lambda_{\max }(Q)\left(R^{2}+U_{\max }^{2}\right)}
$$


The inequality above becomes an equality when $\delta=\delta\left(z\left(t_{k}\right)\right)$, giving the stated result.

Consistent with the observation in Remark 11, we see that $\delta\left(z\left(t_{k}\right)\right) \rightarrow 0$ as $z\left(t_{k}\right) \rightarrow z^{c}$. For an analytic test on the update period $\delta$, we can combine the equation for $\delta\left(z\left(t_{k}\right)\right)$ in Lemma 5 and equation (12) for $\xi$, to obtain

$$
\delta\left(z\left(t_{k}\right)\right)=\frac{(\gamma-1)\left\|z\left(t_{k}\right)-z^{c}\right\|_{Q}^{2}}{\xi+\gamma \lambda_{\max }(Q)\left(R^{2}+U_{\max }^{2}\right)} .
$$

Note that equation for $\delta\left(z\left(t_{k}\right)\right)$ in Lemma 5 is made slightly more conservative here by replacing $(\gamma-1)$ with $\gamma$ in the denominator term. This is done only to facilitate analysis that follows.

Remark 12. The upper bound on the update period in equation (16) has some interesting features. The bound is relatively independent of $\gamma$, particularly if $\gamma \gg 1$. The larger the allowable bounds on the state and control $\left(R\right.$ and $\left.U_{\max }\right)$, and the larger the horizon time $T$, the smaller the required update time. With regard to the control compatibility constraint, i.e. that the optimized control deviate from the assumed control with a norm value at most $\delta^{2} \kappa$, the update must also be faster for larger values of $\kappa$. Given the conservative nature of the proofs, it is not wise to infer too much from the bound in equation (16), but it is reassuring to observe such intuitive affects. Additional comments on the conservatism of the result will be stated at the end of this section.

We also note that since $\delta\left(z\left(t_{k}\right)\right)$ depends on $\left\|z\left(t_{k}\right)-z^{c}\right\|_{Q}^{2}$, a centralized computation is required to generate equation (16) at each receding horizon update. Otherwise, a distributed consensus algorithm, as given in [24] for example, must be run in parallel to determine $\left\|z\left(t_{k}\right)-z^{c}\right\|_{Q}^{2}$, or a suitable lower bound on it. In the dual-mode version defined below, no such centralized computation is required on-line, since a fixed bound on the update period is computed off-line and applied for every receding horizon update.

The first main theorem of the paper is now given.

Theorem 2. Under Assumptions 1 and 3-5, for a given fixed horizon time $T>0$ and for any state $z\left(t_{-1}\right) \in Z_{\Sigma}$ at initialization, if the update time $\delta$ satisfies $\delta \in\left(0, \delta\left(z\left(t_{k}\right)\right)\right], k \in \mathbb{N}$, where $\delta\left(z\left(t_{k}\right)\right)$ is defined in equation (16), then $z^{c}$ is an asymptotically stable equilibrium point of the closed-loop system (8) with region of attraction $Z_{\Sigma}$, an open and connected set.

Proof. If $z\left(t_{k}\right)=z^{c}, \hat{u}_{i}\left(\tau ; z_{i}\left(t_{k}\right)\right)=0$ for all $\tau \in\left[t_{k}, t_{k}+T\right]$ and every $i=1, \ldots, N_{a}$, then the optimal solution to Problem 2 is $u_{\text {dist }}^{*}\left(\tau ; z_{i}\left(t_{k}\right)\right)=0$ for all $\tau \in\left[t_{k}, t_{k}+T\right]$. This is shown in the proof of Proposition 2. Since $A z^{c}=0, z^{c}$ is an equilibrium point of the closed-loop system (8).

We observe that $J_{\Sigma}^{*}\left(z\left(t_{k}\right), T\right)$ has the following properties

- $J_{\Sigma}^{*}\left(z^{c}, T\right)=0$ and $J_{\Sigma}^{*}\left(z\left(t_{k}\right), T\right)>0$ for $z\left(t_{k}\right) \neq z^{c}$,

- $J_{\Sigma}^{*}\left(z\left(t_{k}\right), T\right)$ is continuous at $z\left(t_{k}\right)=z^{c}$, 
- along the trajectory of the closed-loop system from $z\left(t_{0}\right)$, where $z\left(t_{0}\right)=z_{\text {dist }}^{*}\left(t_{0} ; z\left(t_{-1}\right)\right)$ for any initial state $z\left(t_{-1}\right) \in Z_{\Sigma}$,

$$
J_{\Sigma}^{*}\left(z\left(t_{m}\right), T\right)-J_{\Sigma}^{*}\left(z\left(t_{k}\right), T\right) \leq-\int_{t_{k}}^{t_{m}}\left\|z_{\text {dist }}^{*}(\tau)-z^{c}\right\|_{Q}^{2} \mathrm{~d} \tau
$$

for integers $k, m$ with $0 \leq k<m \leq \infty$.

The first two properties follow from Proposition 2. The last property is derived as follows. Combining Lemma 4 and Lemma 3, we have

$$
J_{\Sigma}^{*}\left(z\left(t_{k+1}\right), T\right)-J_{\Sigma}^{*}\left(z\left(t_{k}\right), T\right) \leq-\int_{t_{k}}^{t_{k+1}}\left\|z_{\text {dist }}^{*}\left(\tau ; z\left(t_{k}\right)\right)-z^{c}\right\|_{Q}^{2} \mathrm{~d} \tau .
$$

Applying this recursively gives the result for any $t_{m}>t_{k}$. Following precisely the steps in the proof of Theorem 1 in [6] from this point gives the stated result.

From equation (16), we observe that $\delta\left(z\left(t_{k}\right)\right) \rightarrow 0$ as $z\left(t_{k}\right) \rightarrow z^{c}$. As a consequence, the control comparison constraint gets tighter, and the communication between neighboring agents must happen with increasing bandwidth, as the agents approach their control objective. To mitigate these problems, we now propose a dual-mode version of the distributed receding horizon control law. The closed-loop system will be equation (8) until all agents are in the interior of their terminal constraint sets, at which point each control is synchronously redefined to be the decoupled linear feedback defined in Assumption 3.

To construct the dual-mode version, we will make use of the monotonicity of $J_{\Sigma}^{*}\left(z\left(t_{k}\right), T\right)$ for guaranteeing invariance properties of the distributed receding horizon control law. In particular, under the conditions of Theorem $2, J_{\Sigma}^{*}\left(z\left(t_{k}\right), T\right)$ monotonically decreases until $z\left(t_{k}\right)=z^{c}$. Therefore, the concatenated state $z\left(t_{k}\right)$ is contracting, in some norm-sense, at each receding horizon update. The control switch must therefore rely on a test on the entire state $z\left(t_{k}\right)$ to guarantee all agents are in their terminal constraint sets. A sufficient test is whether

$$
z\left(t_{k}\right) \in \widehat{\Omega}\left(\varepsilon_{\min }\right):=\left\{z \in \mathbb{R}^{2 n N_{a}}:\left\|z-z^{c}\right\|_{\widehat{P}}^{2} \leq \varepsilon_{\min }, \varepsilon_{\min }=\min _{i} \varepsilon_{i}\right\} .
$$

If this holds, then

$$
\left\|z\left(t_{k}\right)-z^{c}\right\|_{\widehat{P}}^{2}=\sum_{i=1}^{N_{a}}\left\|z_{i}\left(t_{k}\right)-z_{i}^{c}\right\|_{P_{i}}^{2} \leq \varepsilon_{\min } \Longrightarrow\left\|z_{i}\left(t_{k}\right)-z_{i}^{c}\right\|_{P_{i}}^{2} \leq \varepsilon_{\min }, \forall i=1, \ldots, N_{a}
$$

guaranteeing all agents are in their terminal constraint sets. Under stated assumptions, we will show that $\left\|z\left(t_{k}\right)-z^{c}\right\|_{W}^{2}$ is contracting with each update, where the positive-definite, symmetric weighting $W$ will be defined more precisely below. Since the contraction is happening with a different norm-weighting than $\widehat{P}$, we require a sufficient test on the $W$-weighted 
quadratic term to guarantee that equation (17) holds. Recall that $\lambda_{\max }\left(Q_{0}\right) \in \mathbb{R}$ is the maximum eigenvalue of any symmetric matrix $Q_{0}$, and let $\lambda_{\min }\left(Q_{0}\right) \in \mathbb{R}$ denote the minimum eigenvalue of $Q_{0}$. Observe that if

$$
\left\|z\left(t_{k}\right)-z^{c}\right\|_{W}^{2} \leq \frac{\lambda_{\min }(W) \varepsilon_{\min }}{\lambda_{\max }(\widehat{P})}
$$

then we have

$$
\lambda_{\min }(W)\left\|z\left(t_{k}\right)-z^{c}\right\|^{2} \leq \frac{\lambda_{\min }(W) \varepsilon_{\min }}{\lambda_{\max }(\widehat{P})} \Longrightarrow\left\|z\left(t_{k}\right)-z^{c}\right\|_{\widehat{P}}^{2} \leq \varepsilon_{\min } .
$$

The test on the $W$-weighted quadratic term is more conservative than testing for equation (17) directly. However, since the $W$-weighted term is shown to strictly decrease for the closed-loop system, we are guaranteed invariance, i.e., once equation (18) is true, it remains true for all future time. Positive invariance is required as it will take some time for the agents to agree, in a distributed way, that they are in the set $\widehat{\Omega}\left(\varepsilon_{\min }\right)$.

It is required that $\delta$ be no larger than a value, denoted $\delta_{\max }$, that guarantees monotonic decrease of the value function $J_{\Sigma}^{*}\left(z\left(t_{k}\right), T\right)$ so that equation (18) holds after some finite time. Now, we assume some quadratic bounds on the function $J_{\Sigma}^{*}$ and show convergence of a sequence such that equation (18), and hence equation (17), is guaranteed to hold after some finite number of iterations, from any feasible state at initialization.

Assumption 6. For any $z \in Z_{\Sigma}$, there exists positive constants $k_{1}, k_{2} \in \mathbb{R}, k_{2}>k_{1}$, and positive-definite, symmetric matrix $W \in \mathbb{R}^{n N_{a} \times n N_{a}}$ such that

$$
k_{1}\left\|z-z^{c}\right\|_{W}^{2} \leq J_{\Sigma}^{*}(z, T) \leq k_{2}\left\|z-z^{c}\right\|_{W}^{2}
$$

where

$$
\begin{aligned}
& \text { - } k_{2}>\delta_{\max } \gamma \lambda_{\min }(Q) / \lambda_{\max }(W) \text { and } \\
& \text { - } k_{2}-k_{1} \leq \delta_{\max } \gamma \lambda_{\min }(Q) /\left(2 \lambda_{\max }(W)\right),
\end{aligned}
$$

and $\delta_{\max }$ is defined as

$$
\delta_{\max }=\frac{(\gamma-1) c}{\xi+\gamma \lambda_{\max }(Q)\left(R^{2}+U_{\max }^{2}\right)}, \quad \text { where } \quad c \triangleq\left(\frac{\lambda_{\min }(W)}{\lambda_{\max }(W)}\right) \frac{\lambda_{\min }(Q) \varepsilon_{\min }}{4 \lambda_{\max }(\widehat{P})} .
$$

The requirement that $k_{2}>\delta_{\max } \gamma \lambda_{\min }(Q) / \lambda_{\max }(W)$ is not too restrictive, since the right-hand side is a small number in general. The requirement that $k_{2}-k_{1} \leq \delta_{\max } \gamma \lambda_{\min }(Q) /\left(2 \lambda_{\max }(W)\right)$ means $k_{2}$ is close to $k_{1}$. Equivalently, this means that $J_{\Sigma}^{*}(z, T)$ basically exhibits quadratic growth, with a $W$-weighted norm. In the analysis that follows, it is not required to know the values of $k_{2}$ and $k_{1}$, so they do not need to be estimated. Since $\delta_{\max }$ is to be computed and used in practice, it will be required to estimate the ratio $\lambda_{\min }(W) / \lambda_{\max }(W)$. More simply, if a reasonable lower bound on this ratio can be computed, the bound can be used to define $c$, although this results in more conservatism. Aside from computing the ratio, or a lower bound on it, the weighting matrix $W$ does not need to be computed. 
Lemma 6. Under Assumptions 1 and 3-6, for a given fixed horizon time $T>0$ and for any state $z\left(t_{-1}\right) \in Z_{\Sigma}$ at initialization, if the update period $\delta=\delta_{\max }$, then for the closed-loop system (8) there exists a finite integer $l \in \mathbb{N}$ at which $z\left(t_{l}\right) \in \widehat{\Omega}\left(\varepsilon_{\text {min }}\right)$ and $z\left(t_{k}\right) \in \widehat{\Omega}\left(\varepsilon_{\text {min }}\right)$ for all $k \geq l$.

Proof. From Lemma 3, the monotonicity statement on $J_{\Sigma}^{*}\left(z\left(t_{k}\right), T\right)$, for all $k \in \mathbb{N}$, is

$$
J_{\Sigma}^{*}\left(z\left(t_{k+1}\right), T\right)-J_{\Sigma}^{*}\left(z\left(t_{k}\right), T\right) \leq-\int_{t_{k}}^{t_{k}+\delta} \sum_{i=1}^{N_{a}} L_{i}^{z}\left(z_{d i}^{*}\left(\tau ; z_{i}\left(t_{k}\right)\right), \hat{z}_{-i}\left(\tau ; z_{-i}\left(t_{k}\right)\right)\right) \mathrm{d} \tau+\delta^{2} \xi .
$$

From the Taylor series approximation in Assumption 5, we have

$$
\begin{aligned}
& \int_{t_{k}}^{t_{k}+\delta} \sum_{i=1}^{N_{a}} L_{i}^{z}\left(z_{d i}^{*}\left(\tau ; z_{i}\left(t_{k}\right)\right), \hat{z}_{-i}\left(\tau ; z_{-i}\left(t_{k}\right)\right)\right) \\
& \quad \approx \delta \gamma\left\|z\left(t_{k}\right)-z^{c}\right\|_{Q}^{2}+\delta^{2} \gamma\left(z\left(t_{k}\right)-z^{c}\right)^{T} Q \dot{z}_{\text {dist }}^{*}\left(t_{k} ; z\left(t_{k}\right)\right) .
\end{aligned}
$$

From the proof of Lemma 5,

$$
-\left(z\left(t_{k}\right)-z^{c}\right)^{T} Q \dot{z}_{\text {dist }}^{*}\left(t_{k} ; z\left(t_{k}\right)\right) \leq \lambda_{\max }(Q)\left(R^{2}+U_{\max }^{2}\right) .
$$

Now, substituting $\delta=\delta_{\max }$, we can rewrite the equation bounding $J_{\Sigma}^{*}\left(z\left(t_{k}\right), T\right)$ as

$$
\begin{aligned}
J_{\Sigma}^{*}\left(z\left(t_{k+1}\right), T\right)-J_{\Sigma}^{*}\left(z\left(t_{k}\right), T\right) & \leq-\delta_{\max } \gamma\left\|z\left(t_{k}\right)-z^{c}\right\|_{Q}^{2}+\delta_{\max }^{2}\left(\xi+\gamma \lambda_{\max }(Q)\left(R^{2}+U_{\max }^{2}\right)\right) \\
& \leq-\delta_{\max } \gamma \frac{\lambda_{\min }(Q)}{\lambda_{\max }(W)}\left\|z\left(t_{k}\right)-z^{c}\right\|_{W}^{2}+\delta_{\max }(\gamma-1) c .
\end{aligned}
$$

Using the bounds on $J_{\Sigma}^{*}\left(z\left(t_{k}\right), T\right)$ from Assumption 6 we have

$$
k_{1}\left\|z\left(t_{k+1}\right)-z^{c}\right\|_{W}^{2} \leq k_{2}\left\|z\left(t_{k}\right)-z^{c}\right\|_{W}^{2}-\delta_{\max } \gamma \frac{\lambda_{\min }(Q)}{\lambda_{\max }(W)}\left\|z\left(t_{k}\right)-z^{c}\right\|_{W}^{2}+\delta_{\max }(\gamma-1) c .
$$

Denoting $y_{k}=\left\|z\left(t_{k}\right)-z^{c}\right\|_{W}^{2} \in[0, \infty)$, we rewrite this as

$$
y_{k+1} \leq \rho y_{k}+\phi, \quad \text { where } \quad \rho=\frac{k_{2}-\delta_{\max } \gamma \lambda_{\min }(Q) / \lambda_{\max }(W)}{k_{1}}, \quad \phi=\frac{\delta_{\max }(\gamma-1) c}{k_{1}} .
$$

Also from Assumption $6, k_{2}>\delta_{\max } \gamma \lambda_{\min }(Q) / \lambda_{\max }(W)$ and

$$
k_{2}-k_{1} \leq \delta_{\max } \gamma \lambda_{\min }(Q) /\left(\lambda_{\max }(W) 2\right)<\delta_{\max } \gamma \lambda_{\min }(Q) / \lambda_{\max }(W),
$$

which implies $0<\rho<1$. Considering the sequence $y_{k+1}$, which is bounded for each $k \in \mathbb{N}$, by $\rho y_{k}+\phi$, we observe that

$$
y_{\infty}=\lim _{k \rightarrow \infty} y_{k+1} \leq \lim _{k \rightarrow \infty}\left[\rho^{k} y_{0}+\phi\left(\sum_{i=0}^{k-1} \rho^{i}\right)\right]=\frac{\phi}{1-\rho} .
$$


Also, we can bound the ratio $\phi /(1-\rho)$ as

$$
\frac{\phi}{1-\rho}=\frac{\delta_{\max }(\gamma-1) c}{k_{1}+\delta_{\max } \gamma \lambda_{\min }(Q) / \lambda_{\max }(W)-k_{2}} \leq \frac{2 \delta_{\max } \lambda_{\max }(W)(\gamma-1) c}{\delta_{\max } \gamma \lambda_{\min }(Q)}<\frac{2 \lambda_{\max }(W) c}{\lambda_{\min }(Q)},
$$

where the first inequality uses the assumed upper bound on $k_{2}-k_{1}$. From the definition of $c$ we have

$$
\frac{2 \lambda_{\max }(W) c}{\lambda_{\min }(Q)}=\frac{\lambda_{\min }(W) \varepsilon_{\min }}{2 \lambda_{\max }(\widehat{P})} .
$$

Therefore, denoting $y_{\infty}=\left\|z\left(t_{\infty}\right)-z^{c}\right\|_{W}^{2}$, we have

$$
\left\|z\left(t_{\infty}\right)-z^{c}\right\|_{W}^{2}<\frac{\lambda_{\min }(W) \varepsilon_{\min }}{2 \lambda_{\max }(\widehat{P})} \Longrightarrow\left\|z\left(t_{\infty}\right)-z^{c}\right\|_{\widehat{P}}^{2}<\frac{\varepsilon_{\min }}{2},
$$

and so $z\left(t_{\infty}\right)$ is in the interior of $\widehat{\Omega}\left(\varepsilon_{\min }\right)$. Moreover, for any $y_{k}=\vartheta+\phi /(1-\rho)$, where $\vartheta \in(0, \infty)$, the sequence bound $y_{k+1} \leq \rho y_{k}+\phi$ guarantees strict monotonic decrease of the sequence. The reason is $\rho y_{k}+\phi-y_{k}=-(1-\rho) y_{k}+\phi=-\vartheta-\phi+\phi=-\vartheta$, and so $y_{k+1} \leq y_{k}-\vartheta$. In particular, once $y_{k} \leq 2 \phi /(1-\rho)$, we are guaranteed that equation (17) holds, since the factor of 2 simply removes the $1 / 2$ in the implication above.

Now, there is a finite integer $l \in \mathbb{N}$ for which $y_{l} \leq 2 \phi /(1-\rho)$. If this were not the case, $y_{k+1} \leq y_{k}-\vartheta$ would hold for all $k \in \mathbb{N}$, which implies $y_{k} \rightarrow-\infty$ as $k \rightarrow \infty$. However, this contradicts the fact that $y_{k} \geq 0$ for all $k \in \mathbb{N}$. Therefore, there exists a finite integer $l \in \mathbb{N}$ for which $y_{l} \leq 2 \phi /(1-\rho)$. Also, since the sequence is required to continue to decrease up to at most the limit bound on $\phi /(1-\rho)$, we have positive invariance as well. This concludes the proof.

Remark 13. If the update period $\delta$ is less than $\delta_{\max }$, then the analysis above still holds, provided the bound on the difference $k_{2}-k_{1}$ in Assumption 6 is tightened by replacing $\delta_{\max }$ by $\delta$.

For the control switch to occur synchronously between the agents, we require a distributed means of determining when equation (18) holds. Since Lemma 6 guarantees monotonic decrease in the $W$-norm sense, we shall cast the test in terms of the $W$-norm. Although this incurs more conservatism, it implies that the agents will not come to agreement unless they have all reached the state for which all subsequent receding horizon updates render $\widehat{\Omega}\left(\varepsilon_{\min }\right)$ a positively invariant set of the closed-loop system.

Distributed Consensus Algorithm for Synchronous Control Switching [24].

The algorithm defined here is a distributed means of determining when equation (18) holds. By distributed, we mean each agent communicates only with neighboring agents. For the algorithm to converge, a minimum number of information exchanges is required. We elect to have a separate sample rate for this algorithm. Over the time interval $\left[t_{k}, t_{k+1}\right)$, we assume neighboring agents will communicate $N_{s}$ times, using notation

$$
\tau_{k, l}=t_{k}+\delta\left(l / N_{s}\right), \quad l=0,1, \ldots, N_{s}-1,
$$


to denote the times at which neighbors exchange information.

To define the dynamics of the switch mechanism, we introduce some notation. At any algorithm update time $\tau_{k, l}$ and for any agent $i$, let $x_{i}\left(\tau_{k, l}\right) \in \mathbb{R}^{+}$be a non-negative scalar value. Also, denote $\varepsilon_{0}=\varepsilon_{\min } / \lambda_{\max }(\widehat{P})$ and $\psi=\lambda_{\max }(W) / \lambda_{\min }(W)$, where $W$ is the weighting matrix defined in Assumption 6. As with the definition of $c$ in Assumption 6, a reasonable upper bound for this ratio, or equivalently a lower bound on $\lambda_{\min }(W) / \lambda_{\max }(W)$, could be used in the place of $\psi$ below. The algorithm is as follows. For every agent $i=1, \ldots, N_{a}$ :

1. At any time $\tau_{k, 0}=t_{k}$, set

$$
x_{i}\left(\tau_{k, 0}\right)=N_{a} \psi\left\|z_{i}\left(t_{k}\right)-z_{i}^{c}\right\|^{2},
$$

transmit $x_{i}\left(\tau_{k, 0}\right)$ and receive $x_{j}\left(\tau_{k, 0}\right)$ from each neighbor $j \in \mathcal{N}_{i}$.

2. For each time $\tau_{k, l}, l=1,2, \ldots, N_{s}-1$,

(a) set

$$
x_{i}\left(\tau_{k, l}\right)=x_{i}\left(\tau_{k, l-1}\right)+\frac{\zeta}{N_{s}} \sum_{j \in \mathcal{N}_{i}}\left(x_{j}\left(\tau_{k, l-1}\right)-x_{i}\left(\tau_{k, l-1}\right)\right),
$$

where $\zeta>0$, and

(b) transmit $x_{i}\left(\tau_{k, l}\right)$ and receive $x_{j}\left(\tau_{k, l}\right)$ from each neighbor $j \in \mathcal{N}_{i}$.

3. Define $x_{i}\left(\tau_{k, N_{s}}\right)$ according to the equation in $2(\mathrm{a})$ above. If

$$
x_{i}\left(\tau_{k, N_{s}}\right) \leq \varepsilon_{0}-\epsilon,
$$

where $\epsilon$ is a specified tolerance satisfying $0<\epsilon \ll \varepsilon_{0}$, then switch at time $t_{k+1}$ and exit the algorithm. Otherwise, return to 1.

\section{End of algorithm.}

Under the conditions stated in a lemma below, namely if $N_{s}$ is sufficiently large, then

$$
\left|x_{i}\left(\tau_{k, N_{s}}\right)-\operatorname{Ave}\left(x\left(t_{k}\right)\right)\right| \leq \epsilon, \quad \forall i=1, \ldots, N_{a},
$$

where

$$
\operatorname{Ave}\left(x\left(t_{k}\right)\right)=\frac{1}{N_{a}} \sum_{i=1}^{N_{a}} x_{i}\left(t_{k}\right)=\psi \sum_{i=1}^{N_{a}}\left\|z_{i}\left(t_{k}\right)-z_{i}^{c}\right\|^{2}=\psi\left\|z\left(t_{k}\right)-z^{c}\right\|^{2} .
$$

From this, we have

$$
\psi\left\|z\left(t_{k}\right)-z^{c}\right\|^{2}-\epsilon \leq x_{i}\left(\tau_{k, N_{s}}\right) \leq \psi\left\|z\left(t_{k}\right)-z^{c}\right\|^{2}+\epsilon .
$$

Therefore, the test in part 3 of the algorithm guarantees that

$$
\left\|z\left(t_{k}\right)-z^{c}\right\|_{W}^{2} \leq \lambda_{\min }(W) \varepsilon_{0}
$$

and equation (18) holds. 
Lemma 7. For a specified tolerance $\epsilon$, satisfying $0<\epsilon \ll \varepsilon_{0}$, the distributed consensus algorithm for synchronous control switching converges in $N_{s}$ iterations provided that

- $N_{s}>\zeta d_{m}$, where $d_{m}$ is the maximum node degree of the graph $\mathcal{G}$, and

- denoting $\lambda=1-\left(\zeta / N_{s}\right) \lambda_{2}(L)$, where $L$ is the Laplacian of the graph $\mathcal{G}$ and $\lambda_{2}(L)$ is the second largest eigenvalue of $L$,

$$
N_{s} \geq \frac{\log \epsilon-\log d_{0}}{\log \lambda}, \quad d_{0}=\left[\sum_{i=1}^{N_{a}}\left(x_{i}\left(\tau_{k, 0}\right)-A v e\left(x\left(t_{k}\right)\right)\right)^{2}\right]^{1 / 2},
$$

where $d_{0}$ denotes a measure of initial disagreement.

It is proven in [24] that the eigenvalue $\lambda_{2}(L)$ bounds the rate of convergence of the average consensus algorithm in continuous time. Converting to discrete time, the convergence bound becomes $\lambda$ defined above, and the first condition in the lemma implies $0<\lambda<1$. The lemma says that if $N_{s}$ is sufficiently large, every agent will meet the tolerance specified in the algorithm. Therefore, the test in step 3 of the algorithm implies the agents agree to synchronously switch to the linear feedback controllers only if equation (18) holds, i.e., if all agents are in the interior of their terminal constraint sets. Note that if $d_{0} \leq \epsilon$, consensus has been reached from the initial values for each $x_{i}$, and the algorithm would terminate in one iteration. The results of the lemma presumes that initially $d_{0}>\epsilon$, as is the case in practice. With $0<\lambda<1$ and $d_{0}>\epsilon$, the second condition on $N_{s}$ provides a lower bound that is positive.

Remark 14. Since $N_{s}$ will be large in general, the communication requirements between receding horizon updates will be demanding for the algorithm to converge. To alleviate this, observe that from the invariance property stated in Lemma 6, we know that once equation (18) holds, it will continue to hold. As such, it is possible to communicate once every receding horizon update. This is done by defining $\tau_{k N_{s}, l}=t_{k N_{s}}+\delta l$, so step 1 is entered every $\delta N_{s}$ seconds. The tradeoff of course is that the algorithm will take considerably more time to converge. A sample rate between these two extremes could be used, one that is appropriate for the given bandwidth limitations.

We now define the dual-mode distributed receding horizon controller.

Definition 10. (Dual-mode distributed receding horizon controller)

Data: Initial state $z\left(t_{-1}\right) \in Z_{\Sigma}$, horizon time $T>0$, update time $\delta=\delta_{\max }$.

Initialization: At time $t_{-1}$, follow the procedure given in Definition 7 , yeiding a control for time $t \in\left[t_{-1}, t_{0}\right]$.

Controller:

1. For $t \in\left[t_{0}, t_{1}\right]$, employ the distributed receding horizon control law $u_{\text {dist }}^{*}\left(t ; z\left(t_{0}\right)\right)$. 
2. At any time $t_{k}, k \in \mathbb{N}$ :

(a) If the distributed consensus algorithm has converged (step 3), employ the decoupled linear feedbacks defined in Assumption 3 for all time $t \geq t_{k}$. Else:

(b) Employ the distributed receding horizon control law $u_{\text {dist }}^{*}\left(t ; z\left(t_{k}\right)\right)$ for time $t \in\left[t_{k}, t_{k+1}\right]$.

Lemma 6 guarantees that, under the assumptions, the inequality in equation (18) will hold after a finite number $l \in \mathbb{N}$ of receding horizon updates. Under the condition of Lemma 7 , the agents will agree to switch at time $t_{l+1}$ to the decoupled linear feedback controllers. Since equation (18) holds, the agents are known to be in a set for which these feedbacks are asymptotically stabilizing. Therefore, the dual-mode distributed receding horizon controller results in asymptotic stability with region of attraction $Z_{\Sigma}$. Formally, we now state this as the second main theorem of the paper.

Theorem 3. Under Assumptions 1 and 3-6 and under the conditions of Lemma 7, for a given fixed horizon time $T>0$ and for any state $z\left(t_{-1}\right) \in Z_{\Sigma}$ at initialization, if the update period $\delta=\delta_{\text {max }}$, then $z^{c}$ is an asymptotically stable equilibrium point of the closed-loop system resulting from the dual-mode distributed receding horizon controller, and $Z_{\Sigma}$ is a region of attraction.

Remark 15. The distributed receding horizon control law of Theorem 2 requires that all agents have the following information available: the horizon time $T$, the update period $\delta$, and all parameters in the computation for $\delta\left(z\left(t_{k}\right)\right)$ in equation (16), which includes the centralized computation of $\left\|z\left(t_{k}\right)-z^{c}\right\|_{Q}^{2}$ at each receding horizon update. The dual-mode distributed receding horizon control law of Theorem 3 requires that all agents have the following information available: the horizon time $T$, all parameters in the computation for $\delta_{\max }$ in Assumption 6, and the parameters for the distributed consensus algorithm, satisfying the conditions in Lemma 7. Clearly, both controllers require some centralized information; however, the dual-mode version does not require any on-line centralized computations. We note that for the controller of Theorem 2, another consensus algorithm could be incorporated for distributed computation of $\left\|z\left(t_{k}\right)-z^{c}\right\|_{Q}^{2}$ at each receding horizon update.

\subsection{Alternative Formulations}

In this section, we discuss two independent modifications to the distributed receding horizon control approach given in Section 4.1. First, we briefly explore the implications of transmitting assumed position information, instead of assumed control information, between neighboring agents. Next, the effects of using a position comparison constraint, rather than the control comparison constraint, in each distributed optimal control problem is investigated.

The distributed optimal control problems here require only the assumed position trajectories from each neighbor. As such, neighboring agents could instead exchange assumed position trajectories, rather than assumed control trajectories. The result would be that agents would then not have to integrate the equations of motion for each neighboring agent, 
and also not require a separate transmission to obtain the initial condition for each neighboring agent. The result is less communication between neighboring agents and simplification in the optimal control computations. In other problems, however, it may be that the state information of neighbors required in each local optimal control problem is more demanding. This would be the case here, for example, if the distributed integrated costs depended also on the assumed velocity of every neighbor. In such cases, the communication requirements are lower by sharing initial state and assumed control trajectory information at each update. To generalize, the tradeoff between exchanging control or state trajectory information, in terms of the overall communication and computation requirements, should dictate how the needed assumed information should be attained.

We now discuss some implications of replacing each control comparison constraint with a position comparison constraint. The control comparison constraint is

$$
\left\|u_{i}\left(s ; z_{i}\left(t_{k}\right)\right)-\hat{u}_{i}\left(s ; z_{i}\left(t_{k}\right)\right)\right\| \leq \delta^{2} \kappa, \quad s \in\left[t_{k}, t_{k}+T\right]
$$

whereas a position comparison constraint is

$$
\left\|q_{i}\left(s ; z_{i}\left(t_{k}\right)\right)-\hat{q}_{i}\left(s ; z_{i}\left(t_{k}\right)\right)\right\| \leq \delta^{2} \kappa, \quad s \in\left[t_{k}, t_{k}+T\right] .
$$

It is the proof of Lemma 3 that requires a bound on the norm of the difference between the assumed position trajectories and the actual position trajectories. If the control comparison constraint is replaced by the position comparison constraint, the result in the lemma still follows by substituting the constraint bounds directly into the bounding argument. The resulting constant $\xi$ is then redefined to be

$$
\xi=\gamma \omega \kappa T(4 M+4 / 3)\left[R+\kappa T^{2}\right] .
$$

Now, $\xi$ grows as a lower-order function of horizon time $T$, with effectively the same growth relation to the other parameters as before. The upper-bound on the update period $\delta$, defined as $\delta_{\max }$ or $\delta\left(z\left(t_{k}\right)\right)$ in equation (16), is proportional to $1 /(\xi+c)$, where $c$ is a constant. Thus, the upper-bound on $\delta$ is potentially larger, i.e., less conservative, for large horizon times, when using the position comparison constraint. Therefore, for a given $\kappa$ and $\delta$ that satisfies the theoretical bounds, the comparison constraint bound $\delta^{2} \kappa$ could be larger using the position comparison constraint, when $T$ is large.

We now discuss another reason that the position comparison constraint may be favorable. Assume that from a given initial condition $z\left(t_{0}\right)$, it takes $N_{0}$ iterations to reach the terminal constraint set using the distributed receding horizon control law, with either control or position comparison constraint. From the proof of Lemma 3, recall that with the control comparison constraint

$$
\left\|q_{d j}^{*}\left(\tau ; z_{j}\left(t_{k}\right)\right)-\hat{q}_{j}\left(\tau ; z_{j}\left(t_{k}\right)\right)\right\| \leq \frac{1}{2} \kappa \delta^{2}\left(\tau-t_{k}\right)^{2} .
$$

As a result we have

$$
\left\|q\left(t_{k+1}\right)-q\left(t_{k}\right)\right\| \leq \frac{\delta^{4} \kappa}{2}, \text { for each } k \in \mathbb{N} \quad \Longrightarrow \quad\left\|q\left(t_{N_{0}}\right)-q\left(t_{0}\right)\right\| \leq N_{0} \frac{\delta^{4} \kappa}{2} .
$$


On the other hand, from the position comparison constraint we have

$$
\left\|q\left(t_{N_{0}}\right)-q\left(t_{0}\right)\right\| \leq N_{0} \delta^{2} \kappa
$$

A reasonable assumption is that $N_{0} \delta$ is a constant, meaning that the number of iterations is proportional to the receding horizon sample rate. The equations above imply that the closedloop position trajectory will deviate from the position trajectory at initialization by a factor of $\delta^{3}$ with the control comparison constraint, and by a factor of $\delta$ with the position comparison constraint. The theory requires the $\delta$ be sufficiently small. Therefore, obeying the sufficient conditions in the theory implies that the receding horizon controller will resemble the initial open-loop solution more when using the control comparison constraint.

Suppose now that the linear, homogeneous vehicle dynamics are replaced by the more generic nonlinear dynamics

$$
\dot{z}_{i}(t)=f_{i}\left(z_{i}(t), u_{i}(t)\right), \quad \text { for each } i=1, \ldots, N_{a}
$$

The dynamics above require additional assumptions, e.g., stabilizability around each respective equilibrium point $z_{i}^{c}$, as specified in the formulation in [6]. Suppose that the cost is still quadratic, with every distributed integrated cost $L_{i}$ depending upon each neighboring state $z_{j}$ (or some components of the state), for $j \in \mathcal{N}_{i}$. Again, the proof of Lemma 3 will require a bound on the norm of the difference between the assumed trajectories $\hat{z}_{i}$ and the actual trajectories $z_{i}^{*}$, for every $i=1, \ldots, N_{a}$. If the comparison constraint is in terms of the state $z_{i}$, then the theoretical results follow immediately, since the constraint bounds can be directly substituted into the bounding argument in the proof. If the control comparison constraint is used, however, achieving a bound on the difference between assumed and actual state trajectories becomes more cumbersome. Finally, we note that the analysis in [6] can be used for construction of the distributed terminal cost and constraint functions.

From a numerical point of view, the control comparison constraint may be more appropriate. Specifically, consider the case where, for a given horizon time $T$, the value of the allowable product $\delta^{2} \kappa$ is not too different for the control and position comparison constraint formulations. The control comparison constraint implies that

$$
\left\|q_{i}\left(s ; z_{i}\left(t_{k}\right)\right)-\hat{q}_{i}\left(s ; z_{i}\left(t_{k}\right)\right)\right\| \leq\left(s-t_{k}\right)^{2} \delta^{2} \kappa / 2, \quad s \in\left[t_{k}, t_{k}+T\right] .
$$

Thus, we have a parabolic constraint on the position, and for $s \geq t_{k}+\sqrt{2}$, this constraint is less stringent than the position comparison constraint. If $T$ is large, then, each distributed optimization problem is generally easier with the control comparison constraint, in the sense that the position trajectory is not constrained to be as close to the previous trajectory, as time proceeds.

The observations above should be taken in the light that the theoretical conditions are conservative. In practice, values for $\delta$ much larger than those specified by the theory are successful. Moreover, the control comparison constraint has been tested in simulations, with success, while the position comparison constraint has yet to be tested. As part of our future work, we shall compare the performance of the two formulations in simulations. 
In the next section, formations of vehicles are stabilized using the centralized and distributed receding horizon controllers defined in the previous sections. The simulations reveal that for a fixed, small value for the update period $\delta$, convergence is obtained with good accuracy. Moreover, the performance of the distributed implementation is comparable to that of the centralized implementation.

\section{Formation Stabilization Example}

A simulation of a four vehicle formation is presented in this section. The dimension of the position vector for all agents is two $(n=2)$. The state and control constraint sets are defined as

$$
\mathcal{Z}=\mathbb{R}^{4}, \quad \mathcal{U}=\left\{\left(u_{1}, u_{2}\right) \in \mathbb{R}^{2}:-1 \leq u_{j} \leq 1, j=1,2\right\}
$$

The objective is a fingertip formation that tracks the reference trajectory $\left(q_{\text {ref }}(t), \dot{q}_{\text {ref }}(t)\right) \in$ $\mathbb{R}^{4}$, defined as

$$
q_{\mathrm{ref}}(t)=\left\{\begin{array}{ll}
(t, 0), & t \in[0,10) \\
(10,10-t), & t \in[10, \infty)
\end{array},\right.
$$

where $t_{0}=0$ in the notation of the previous sections. The acceleration $\ddot{q}_{\text {ref }}(t)$ is zero for all time except at $t=10$ seconds. To be consistent with the cooperative stabilization objective, we rewrite the system dynamics in equation (1) in error form. In particular, the error system for any agent $i$ has state $\left(q_{i}-q_{\text {ref }}, \dot{q}_{i}-\dot{q}_{\text {ref }}\right)$ and dynamics $\ddot{q}_{i}=u_{i}$. The jump in the reference velocity at time $t=10$ serves to examine how well the error dynamics are stabilized for two different legs of the reference trajectory. Equivalently, the problem is to stabilize the error dynamics from an initial condition at time 0 , and then again from the current state at time 10 .

To eliminate any offset between the center of geometry of the formation and the reference trajectory, we set the formation path to $q_{d}(t)=(0,0)$ for all $t \geq 0$. The vector formation graph is defined by vertices $\mathcal{V}=\{1,2,3,4\}$ and relative vectors $\mathcal{E}=\{(1,2),(1,3),(2,4)\}$. As in the generalization of Section 2, the core vehicles associated with the tracking cost are $\{1,2,3\}$. The relative vectors are defined for the two legs of the reference trajectory as

$$
d_{12}=d_{24}=\left\{\begin{array}{ll}
(-2,1), & t \in[0,10) \\
(1,2), & t \in[10, \infty)
\end{array} \quad, \quad d_{13}=\left\{\begin{array}{ll}
(-2,-1), & t \in[0,10) \\
(-1,2), & t \in[10, \infty)
\end{array} .\right.\right.
$$

The common rotation in the vectors at time $t=10$ is match the heading of the fingertip formation with the heading of the reference trajectory. The initial conditions for each agent are given as $q_{1}(0)=(-1,2), q_{2}(0)=(-4,0), q_{3}(0)=(-2,0)$ and $q_{4}(0)=(-7,-1)$, with $\dot{q}_{i}(0)=(0,0)$ for each agent $i \in \mathcal{V}$. In both centralized and distributed receding horizon implementations, a horizon time of $T=5.0$ is used. Also, the following weighting parameter values are consistent in both implementations: $\omega=2.0, \nu=1.0$ and $\mu=2.0$. As stated, collision avoidance is not incorporated in the optimal control problems, either by cost or constraint. In all simulation results presented, no collisions were observed to occur. 
To solve the optimal control problems numerically, we employ the Nonlinear Trajectory Generation (NTG) software developed at Caltech. A detailed description of NTG as a realtime trajectory generation package for constrained mechanical systems is given in [21]. The package is based on finding trajectory curves in a lower dimensional space and parameterizing these curves by B-splines. Sequential quadratic programming (SQP) is used to solve for the B-spline coefficients that optimize the performance objective, while respecting dynamics and constraints. The package NPSOL [12] is used to solve the SQP problem.

For the centralized receding horizon control law, parameter values in the optimal control problem must be chosen to guarantee that Assumption 2 is true. For the weights chosen above, $K$ is defined as the linear quadratic regulator and $P$ the corresponding stable solution to the algebraic Riccati equation. Choosing $\alpha=0.4$ implies that the assumption (i) is true. To prove this, define $y=z-z^{c}$ and observe that

$$
\begin{aligned}
y^{T} P y & \leq \alpha \quad \Leftrightarrow \quad \lambda_{\max }(P) y^{T} P y \leq \lambda_{\max }(P) \alpha \quad \Rightarrow \quad y^{T} P^{2} y \leq \lambda_{\max }(P) \alpha \\
& \Rightarrow \quad y^{T} P B B^{T} P y \leq \lambda_{\max }(P) \alpha \quad \Leftrightarrow \quad y^{T} K^{T} K y \leq \frac{\lambda_{\max }(P) \alpha}{\mu^{2}} .
\end{aligned}
$$

Choosing $\alpha \leq \mu^{2} / \lambda_{\max }(P) \approx 0.4$ guarantees that $\|K y\|^{2} \leq 1$. Finally, the latter condition guarantees that $K\left(z-z^{c}\right) \in \mathcal{U}^{N_{a}}$ for all $z \in \Omega(\alpha)$, since each component of $K y$ will be between -1 and 1 for all time. For an update period of $\delta=0.5$, centralized receding horizon control of the fingertip formation is shown in Figure 2. The four closed-loop position trajectories of the

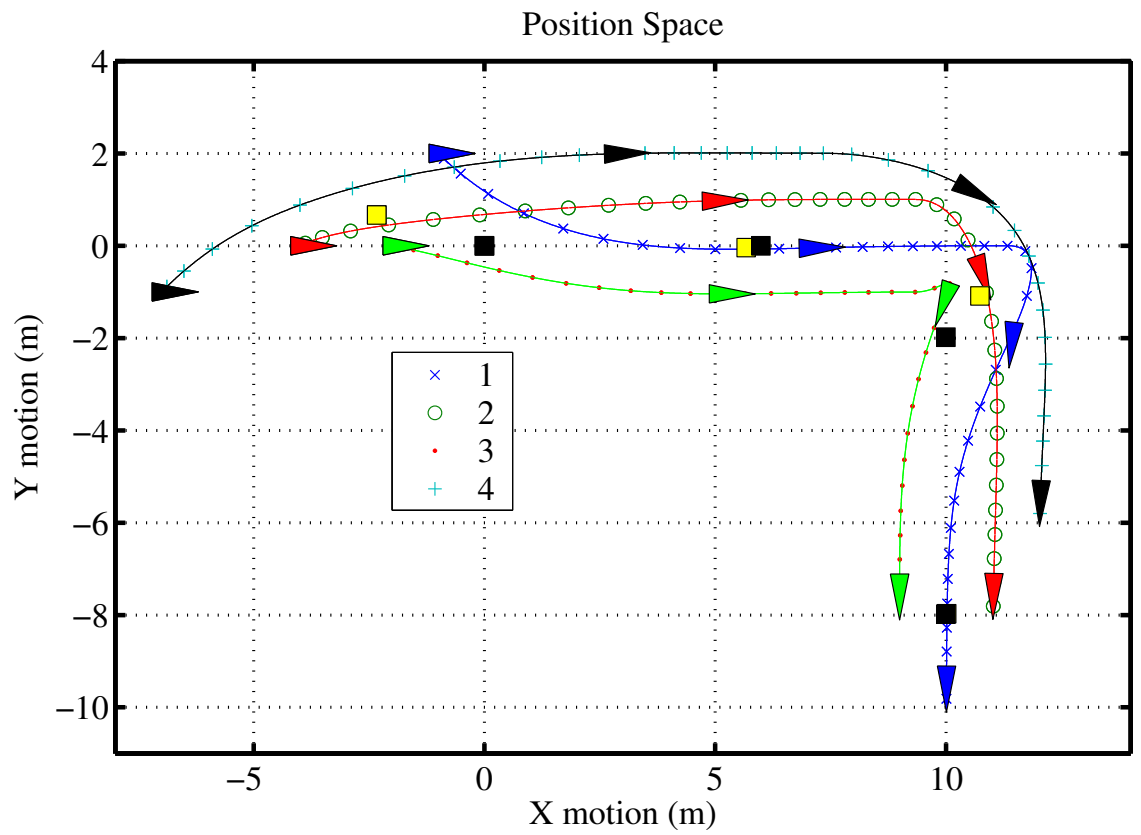

Figure 2: Four vehicle fingertip formation using centralized receding horizon control. vehicles are shown in the figure, with each vehicle depicted by a triangle. The heading of any 
triangle shows the direction of the corresponding velocity vector. The symbols along each trajectory mark the points at which the receding horizon updates occur. The legend identifies a symbol with a vehicle number for each trajectory. The triangles show the position and heading of each vehicle at snapshots of time, specifically at 0.0, 6.0, 12.0 and 18.0 seconds.

Also shown at these instants of time are the reference trajectory position $q_{\text {ref }}(t)$, identified by the black square, and the average position of the core vehicles $q_{\Sigma}(t)$, identified by the yellow square. The tracking part of the cooperative objective is achieved when $q_{\Sigma}(t)=q_{\text {ref }}(t)$, i.e., when the two squares are perfectly overlapping. At time 6.0, the vehicles are close to the desired formation, and the squares are nearly overlapped, indicating that the tracking objective is being reached. After 8.0 seconds, the formation objective has been met to a numerical precision of 0.01 , which is the value of the the optimal cost function at that time. At time 12.0, the snapshot shows the formation reconfiguring to the change in heading of the reference trajectory which occurred at time 10.0. At time 18.0, the objective has again been met and the optimal cost function has a numerical value of less that 0.01.

The receding horizon control law time history for agent 3 is shown in Figure 3 . At receding

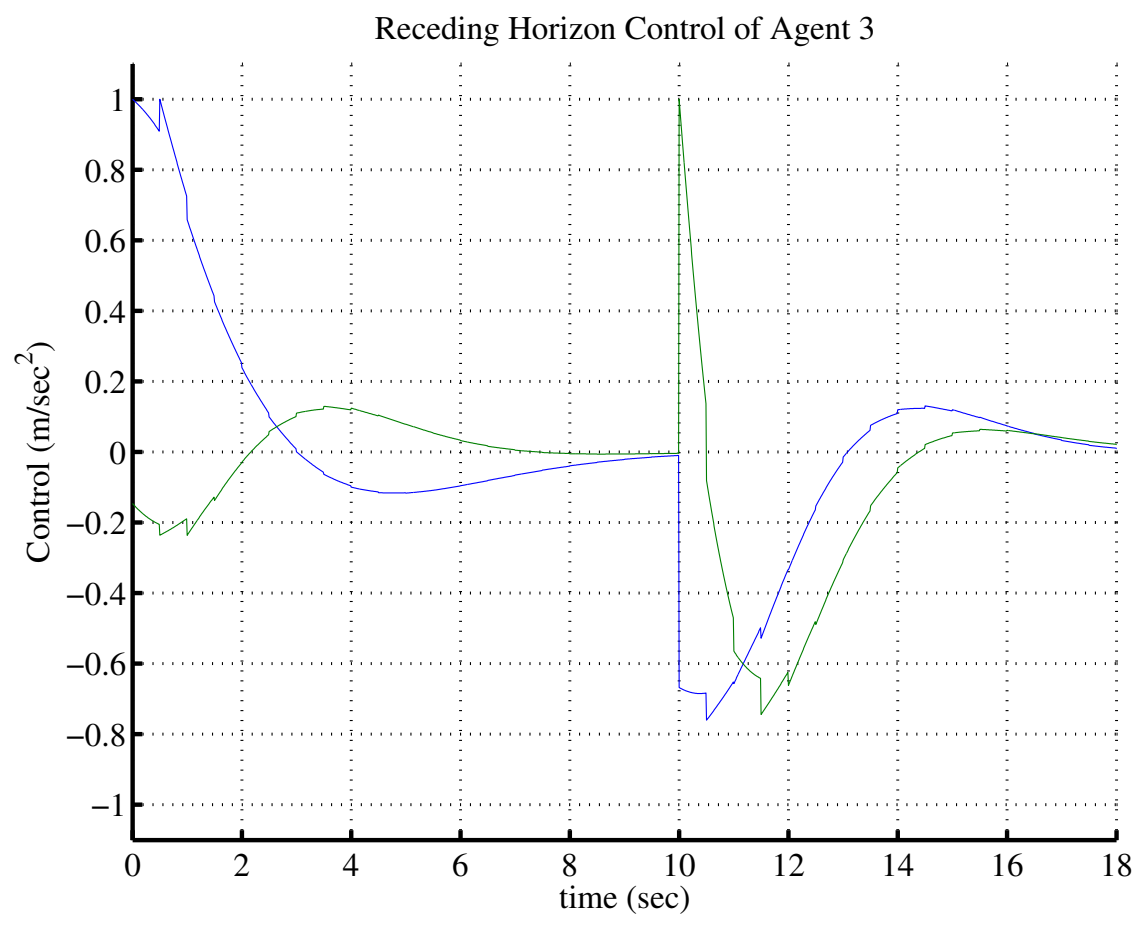

Figure 3: Centralized receding horizon control law time history for agent 3.

horizon updates, the control is not required to initially match the last control value applied. Consequently, the resulting closed-loop control will be discontinuous in general. The figure shows greater discontinuity during the transient phase of the closed-loop response, with the largest discontinuity occurring at time 0.0 and at time 10.0, when the reference trajectory changed heading. 
For the distributed receding horizon implementation, the initial state at time 0.0 is used for initialization, as described in Definition 7. In terms of the notation, we thus have $t_{-1}=$ 0.0. Regarding the conditions in Assumption 3, we first choose $Q_{i}=\lambda_{\max }(Q) I_{(4)}$, where $\lambda_{\max }(Q) \approx 6.85$. As in the centralized case, $K_{i}$ is defined as the linear quadratic regulator and $P_{i}$ the corresponding stable solution to the algebraic Riccati equation. Following the steps above, we can show that $\alpha_{i}=0.33$ guarantees that the conditions in the assumption will hold. Finally, we set $\gamma=2$ in the cost functions of the distributed optimal control problems. The distributed receding horizon controller is applied for all time and switching to the decoupled feedbacks is not employed.

Before employing the distributed receding horizon control law define in Section 4.1, we first explore what happens when neighbors are assumed to have zero control and the control comparison constraint is not enforced $(\kappa=+\infty)$. This was explored in simulations in a previous paper [10]. In other words, neighbors are assumed to continue along straight line paths over any optimization horizon. For an update period of $\delta=0.5$, the result is shown in Figure 4. The receding horizon control law time history for agent 3 is shown in Figure 5.

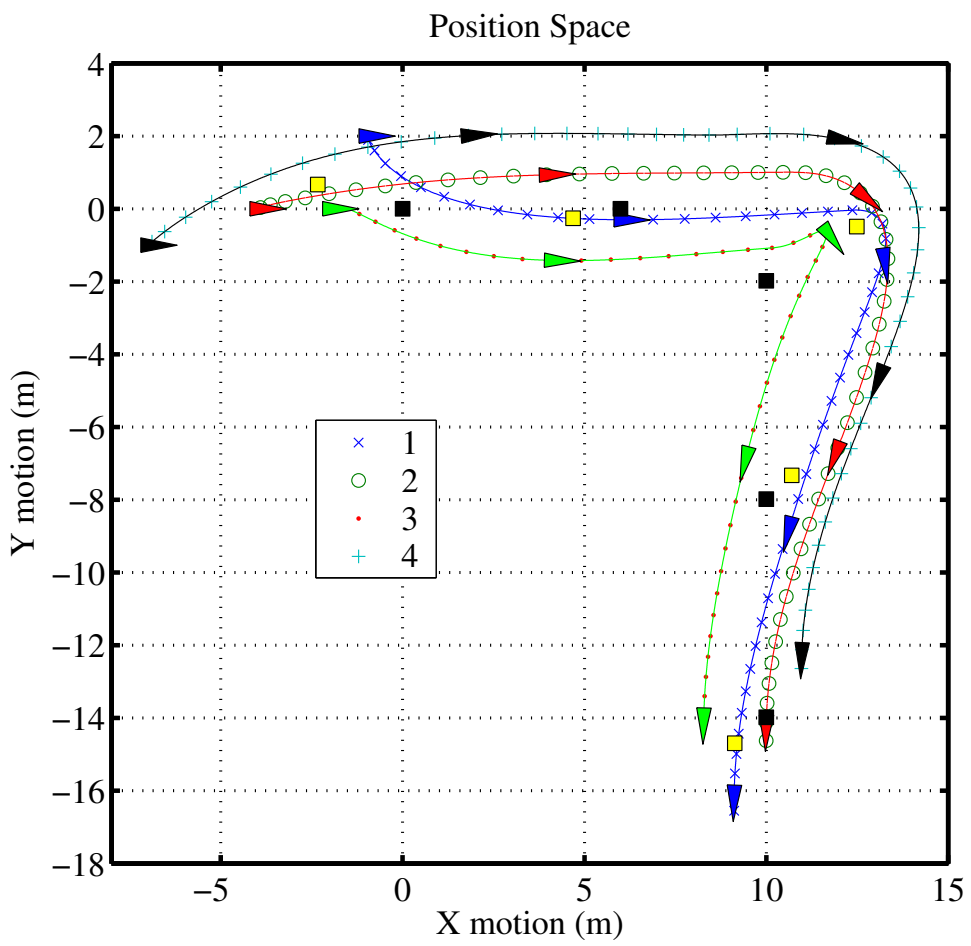

Figure 4: Four vehicle formation using distributed receding horizon control, assuming neighbors continue along straight line paths at each update and without enforcing the control comparison constraints $(\kappa=+\infty)$.

The response is characterized by overshoot, as agents believe neighbors will continue along vectors tangent the path over the entire optimization horizon at each update. The figure 

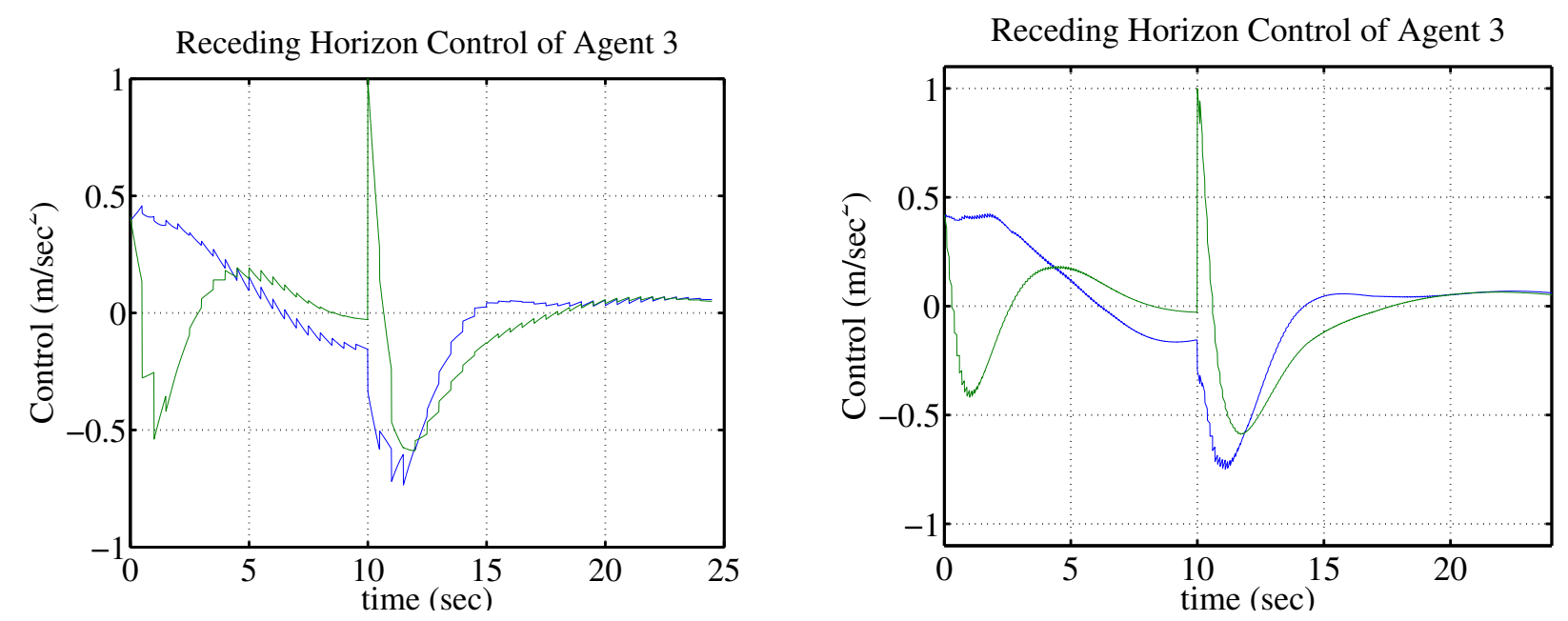

Figure 5: Distributed receding horizon control law time history of agent 3. Left figure shows time history when $\delta=0.5$, and right figure shows time history when $\delta=0.1$.

shows the position and heading triangles at the same snapshots of time as before, plus one additional snapshot at time 24.0. As time grows, the formation is observed to get closer to meeting the formation objective, but only after a long time. Moreover, the formation never meets the objective to acceptable precision.

Interestingly, for this given weights in the cost function, the closed-loop performance is observed to stay the same even if the update period is decreased. In particular, if $\delta$ is reduced to 0.25 or 0.1 , the response is nearly the same. For example, the receding horizon control law time history for agent 3 with $\delta=0.1$ is also shown in Figure 5 . As in the previous paper [10], other parameters were observed to improve the performance in this case. Specifically, increasing the damping weighting $\nu$ reduces the overshoot effect, although the response of the formation naturally becomes more sluggish. Also, if the horizon time $T$ is shortened, overall performance improves, as the assumption becomes more valid. The reason is that a straight line approximation is generally a valid approximation locally, and shrinking $T$ means the assumption should hold over a more local domain, relative to larger values of $T$.

In the formulation in [16], where each agent optimizes for itself as well as for neighboring agents, a similar effect is observed. There, agents assume that neighbors will react solely with regard to the local cost function and constraints. Apparently, such a self-interested philosophy is not too bad if agents are not looking too far into the future, since initial conditions are consistent in all distributed optimization problems at each update.

Now, we consider the performance of the distributed receding horizon control law defined in Section 4.1. After initialization, the control comparison constraint is enforced, setting $\kappa=2$. Although the norm in each control comparison constraint is defined to be the Euclidean 2-norm, we implement the $\infty$-norm, since it is a linear constraint and therefore easier for the optimization algorithm. For an update period of $\delta=0.5$, centralized receding horizon control of the fingertip formation is shown in Figure 6. As before, the triangles show the position and heading of each vehicle at the time snapshots of 0.0, 6.0, 12.0 and 18.0 


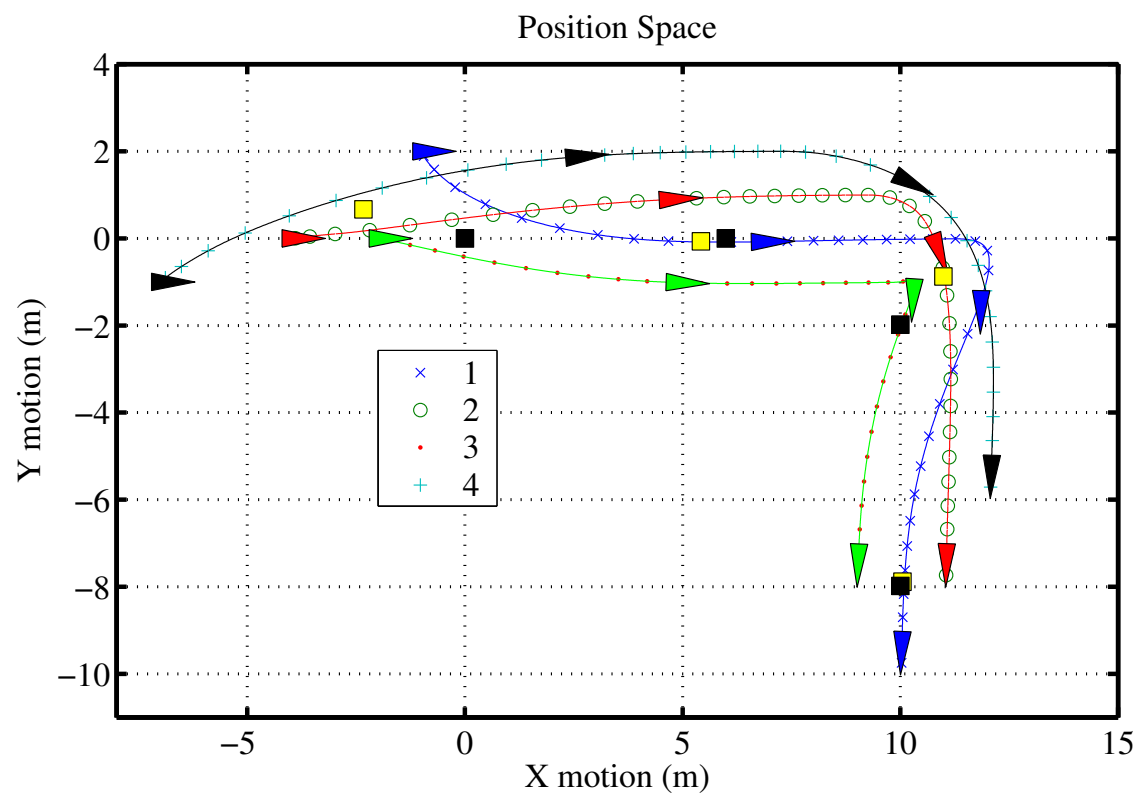

Figure 6: Four vehicle formation using distributed receding horizon control.

seconds. The performance is close to that of the centralized implementation. At snapshot time 6.0, the formation is slightly lagging the reference, compared to the centralized version. Also, vehicles 1 and 3 in particular slightly overshoot, in comparison to their centralized counterparts, when the reference heading changes at time 10.0. At time 18.0, the formation objective is close to being met, and for slightly more time the same precision as the centralized implementation is achieved. The distributed receding horizon control law time history for agent 3 is shown in Figure 7. In the control comparison constraint, we have that $\delta^{2} \kappa=$ 0.5. As a byproduct of this constraint, the allowable size of discontinuity in the closedloop control at receding horizon update times is reduced. In particular, for the centralized implementation, the control can jump by as much as 2, e.g., from -1 to 1 . However, in the distributed implementation with the control comparison constraint, since we used the $\infty$-norm, each component of the control can jump by 0.5 at most. The control in Figure 7 shows a few places where such maximal discontinuities occur, specifically just after time 10.0 when the reference trajectory changes heading.

Since agents are relying on the assumption that neighbors keep doing what they were doing, and the control comparison constraint ensures that the assumption is not too far off, stability is ensured. In fact, if the comparison constraint is removed, stability is observed in the simulation for the chosen parameter values above. The sensitivity to horizon time, as observed above when neighbors are assumed to continue along straight-line paths, and as observed in the formulation in [16], is no longer present.

Regarding the communication requirements of transmitting assumed controls to neighboring agents, in the NTG formulation corresponding to the simulations above, 14 B-spline 


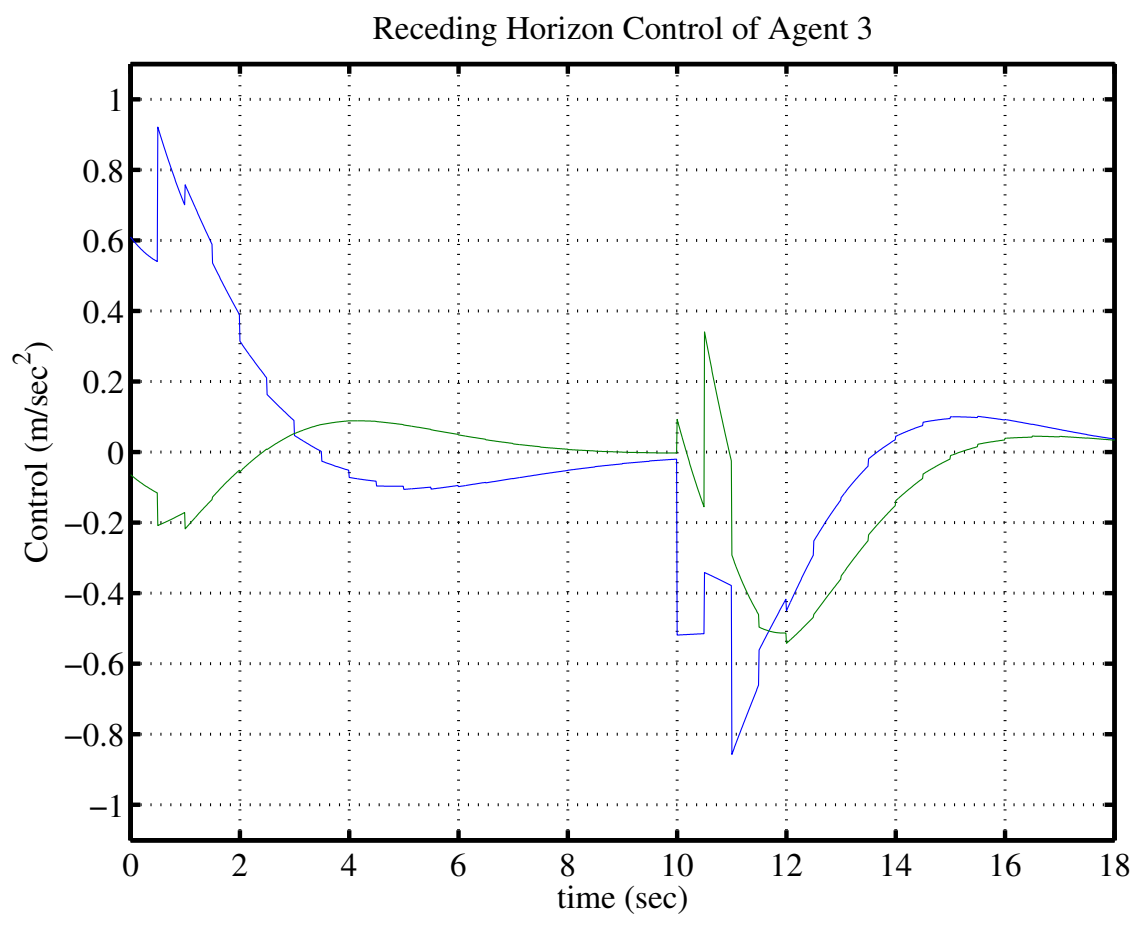

Figure 7: Distributed receding horizon control law time history for agent 3.

coefficients specified the two-dimensional assumed control trajectory of each agent. In comparison, when agents assume neighbors continue along straight lines, 4 numbers much be communicated at each update, representing the initial condition of the state at the update time. Thus, such representations of trajectories in the optimization problem can aid in keeping the communication requirements closer to that of other decentralized schemes [8].

\section{Conclusions and Extensions}

We have shown under what assumptions a centralized optimal control problem, whose cost couples the states of a set of dynamically decoupled subsystems, could be decomposed into a set of distributed optimal control problems for a distributed receding horizon implementation. The implementation requires an additional constraint in the local optimal control problems, namely a constraint ensuring that assumed and applied control trajectories not deviate too far from one another. Asymptotic stability is proven in the absence of uncertainty and for sufficiently fast receding horizon updates. Although the details of the theory here are specific to homogeneous and linear dynamics, the approach is general and the nonlinear, inhomogeneous case is worked out elsewhere [9].

As discussed in Section 4.3, the theory is quite conservative in that, when the update period is small enough to satisfy the theoretical conditions for asymptotic stability, the 
control comparison constraint implies the closed-loop trajectories must remain close to the trajectories computed at initialization. This implication is not a desirable property, as it takes away from the power of the receding horizon philosophy, namely, the ability to recompute an optimal action based on current conditions. Still, in practice, larger values for the update period than required by the theory achieve performance that is comparable to the centralized implementation. Less conservative results will be one objective in our ongoing research.

The inter-agent communication requirements of our approach are more intensive than that of decentralized feedback control [8]. The tradeoff is that the power of an optimizationbased approach is available, namely, the ability to address performance in the presence of generic constraints and nonlinearities. Moreover, no particular structure in the overall system dynamics is required; all agents could have different dynamics. We also note that the dimension of each distributed optimal control problem is equal to that of an optimal control problem of the single corresponding agent. Thus, there is considerable improvement in tractability over the centralized problem, particularly when the number of agents $N_{a}$ is large. If the trajectories are known to be sufficiently smooth, and polynomial-based approximations are valid, the communication requirements need not be substantially worse than that of standard decentralized schemes.

We should also emphasize that the multi-vehicle formation stabilization problem is simply a venue. In other problems where the performance objective, specifically the integrated cost, is decomposable in such a way that the summation recovers the centralized cost, the approach is applicable. Also, if constraints that couple the states and/or controls of neighboring agents are present, the distributed implementation still preserves the property that if there exists a feasible solution at initialization, there exits a feasible solution to all subsequent distributed optimizations problems. The initialization phase of the distributed implementation, however, now requires a better guess for the assumed controls, namely, one that is known to be feasible. Still, this requirement is no stronger than that imposed on the centralized implementation; if the centralized problem has an initial feasible solution, subsequent feasibility can be ensured. If an initially feasible solution to the centralized problem is available, it could be used to define the assumed controls at initialization in the distributed case. For the asymptotic stability results to hold, the coupling constraints must satisfy the stated conditions on the set $\mathcal{Z}$. It may be of interest to see if coupling in the dynamics, as with the problem addressed in [14], could also be addressed by the formulation here.

The theory will ultimately be applied to the Caltech Multi-Vehicle Wireless Testbed [7]. Other venues for application of the theory may exist, for example, in dynamic formulations of resource allocation problems in networks, or in dynamic game theoretic settings. For instance, the approach by Baglietto et al [2] for distributed dynamic routing in a network could be compared to a discrete-time version of our distributed receding horizon control law. 


\section{Acknowledgments}

The authors gratefully acknowledge Prof. Jeff Shamma, Dr. Reza Olfati Saber and Prof. Nicolas Petit for their help in preparing and revising this work.

\section{References}

[1] L. Acar. Boundaries of the receding horizon control for interconnected systems. Journal of Optimization Theory and Applications, 84(2), 1995.

[2] M. Baglietto, T. Parisini, and R. Zoppoli. Neural approximations and team theory for dynamic routing: A receding horizon approach. In Proceedings of the IEEE Conference on Decision and Control, Phoenix, AZ, 1999.

[3] D. P. Bertsekas and J. N. Tsitsiklis. Parallel and Distributed Computation: Numerical Methods. Athena Scientific, 1997.

[4] B. Bollobás. Modern Graph Theory. Graduate Texts in Mathematics, Springer-Verlag, 1998.

[5] H. Chen. Stability and Robustness Considerations in Nonlinear MPC. PhD thesis, University of Stuttgart, 1997.

[6] H. Chen and F. Allgöwer. A quasi-infinite horizon nonlinear model predictive scheme with guaranteed stability. Automatica, 14(10):1205-1217, 1998.

[7] L. Cremean, W.B. Dunbar, D. van Gogh, J. Meltzer, R.M. Murray, E. Klavins, and J. Hickey. The Caltech multi-vehicle wireless testbed. In Proceedings of the Conference on Decision and Control, Las Vegas, NV, 2002.

[8] R. D'Andrea and G. E. Dullerud. Distributed control design for spatially interconnected systems. Accepted for publication, IEEE Transactions on Automatic Control, 2003.

[9] W. B. Dunbar. Distributed Receding Horizon Control of Multi-Agent Systems: Theoretical and Numerical Algorithms. PhD thesis, California Institute of Technology, In Preparation: 2004.

[10] W. B. Dunbar and R. M. Murray. Model predictive control of coordinated multi-vehicle formations. In Proceedings of the IEEE Conference on Decision and Control, Las Vegas, NV, 2002.

[11] J. Alexander Fax and Richard M. Murray. Graph laplacians and stabilization of vehicle formations. In Proceedings of the IFAC World Congress, Barcelona, Spain, 2002. 
[12] P. Gill, W. Murray, M. Saunders, and M. Wright. User's guide for NPSOL 5.0: A fortran package for nonlinear programming. Systems Optimization Laboratory, Stanford University, Stanford, CA 94305, 1998.

[13] G. Grimm, M. J. Messina, S. E. Tuna, and A. R. Teel. Example of zero robustness in constrained model predictive control. In Proceedings of the IEEE Conference on Decision and Control, Maui, HI, 2003.

[14] D. Jia and B. H. Krogh. Distributed model predictive control. In Proceedings of the American Control Conference, 2001.

[15] D. Jia and B. H. Krogh. Min-max feedback model predictive control for distributed control with communication. In Proceedings of the American Control Conference, 2002.

[16] T. Keviczky, F. Borrelli, and G. J. Balas. Model predictive contorl for decoupled systems: A study of decentralized schemes. In Submitted to the American Control Conference, Boston, MA, 2004.

[17] N. E. Leonard and E. Fiorelli. Virtual leaders, artificial potentials and coordinated control of groups. In 2001 Conference on Decision and Control, Florida, 2001.

[18] D.Q. Mayne, J.B. Rawlings, C.V. Rao, and P.O.M. Scokaert. Contrained model predictive control: Stability and optimality. Automatica, 36:789-814, 2000.

[19] M. Mesbahi and F. Y. Hadaegh. Formation flying control of multiple spacecraft via graphs, matirx inequalities, and switching. Journal of Guidance, Control and Dynamics, 24(2):369-377, 2001.

[20] H. Michalska and D.Q. Mayne. Robust receeding horizon control of contrained nonlinear systems. IEEE Trans. Auto. Contr., 38:1623-1632, 1993.

[21] M. B. Milam, K. Mushambi, and R. M. Murray. A new computational approach to real-time trajectory generation for constrained mechanical systems. In Proceedings of the Conference on Decision and Control, 2000.

[22] N. Motee and B. Sayyar-Rodsari. Optimal partitioning in distributed model predictive control. In Proceedings of the American Control Conference, 2003.

[23] R. Olfati-Saber, W. B. Dunbar, and R. M. Murray. Cooperative control of multi-vehicle systems using cost graphs and optimization. In Proceedings of the American Control Conference, Denver, CO, 2003.

[24] R. Olfati Saber and R. M. Murray. Consensus Problems in Networks of Agents with Switching Topology and Time-Delays. submitted to the IEEE Trans. on Automatic Control, 2003. 
[25] W. Ren and R.W. Beard. A decentralized scheme for spacecraft formation flying via the virtual structure approach. Journal of Guidance, Control and Dynamics, To Appear. Revised Submission: June, 2003.

[26] A. G. Richards, J. P. How, T. Schouwenaars, and E. Feron. Spacecraft trajectory planning with avoidance constraints using mixed-integer linear programming. AIAA Journal of Guidance Control and Dynamics, 25(4):755-764, 2002. 